\title{
Clinical applications of dendritic cells-cytokine- induced killer cells mediated immunotherapy for pancreatic cancer: an up-to-date meta-analysis
}

This article was published in the following Dove Press journal:

OncoTargets and Therapy

23 August 2017

Number of times this article has been viewed

\section{Yucai Zhang' \\ Xiaorui Zhang' \\ Anqi Zhang ${ }^{2}$ \\ $\mathrm{Ke} \mathrm{Li}^{2}$ \\ Kai $\mathrm{Qu}^{3}$}

'Department of Health, ${ }^{2}$ Department of Central Laboratory, Liaocheng People's Hospital of Taishan Medical University, Liaocheng, Shandong, ${ }^{3}$ Department of Hepatobiliary Surgery, The First Affiliated Hospital of Xi'an Jiaotong University, Xi'an, Shaanxi, China
Correspondence: Kai Qu

Department of Hepatobiliary Surgery, The First Affiliated Hospital of Xi'an Jiaotong University, No 277 West Yan-ta Road, Xi'an 71006I, Shaanxi, China Tel +86 I36 09| I 7I04 Emailxajtquk@।26.com

$\mathrm{Ke} \mathrm{Li}$

Department of Central Laboratory, Liaocheng People's Hospital of Taishan Medical University, Dongchang West Road, No 67, Liaocheng 252000,

Shandong, China

Tel +86 I52 63565765

Email zxsyslk@l63.com
Purpose: This study aimed to systematically evaluate the efficacy and safety of dendritic cells-cytokine-induced killer (DC-CIK) cells immunotherapy in treating pancreatic cancer (PC) patients.

Methods: Data were collected from published articles of clinical trials. Databases including Web of Science, EMBASE, PubMed, Cochrane Library, Wanfang, and CNKI were searched. The main outcome measures in this research included the overall response rate (ORR), disease control rate (DCR), overall survival (OS), patients' quality of life (QoL), immune function, and adverse events. Comparative analysis was conducted between DC-CIK immunotherapy and chemotherapy (combined therapy) and chemotherapy alone.

Results: This analysis covered 14 trials with 1,088 PC patients involved. The combined therapy showed advantages over chemotherapy alone in ORR (odds ratio $[\mathrm{OR}]=1.69,95 \%$ confidence interval $[\mathrm{CI}]=1.20-2.38, P=0.003)$, DCR (OR $=2.33,95 \% \mathrm{CI}=1.63-3.33, P<0.00001)$, OS $(1$-year OS, OR $=3.61,95 \%$ CI $=2.41-5.40, P<0.00001 ; 3$-year OS, OR $=2.65,95 \%$ $\mathrm{CI}=1.56-4.50, P=0.0003)$ and patients' $\mathrm{QoL}(P<0.01)$ with statistical significance. After immunotherapy, lymphocyte subsets' percentages of $\mathrm{CD}^{+}(P<0.00001), \mathrm{CD}^{+}(P=0.01), \mathrm{CD}^{+} \mathrm{CD}^{+} 6^{+}$ $(P<0.00001)$, and cytokine levels of IFN- $\gamma(P<0.00001)$ were significantly increased, and the percentages of $\mathrm{CD} 4{ }^{+} \mathrm{CD} 25^{+} \mathrm{CD} 127^{\text {low }}(P<0.00001)$ and levels of IL-4 $(P<0.0001)$ were significantly decreased, whereas analysis on $\mathrm{CD}^{+}(P=0.59)$ and $\mathrm{CD}^{+} / \mathrm{CD} 8^{+}$ratio $(P=0.64)$ did not show a significant difference.

Conclusion: The combination of DC-CIK immunotherapy and chemotherapy is effective for PC treatment, indicated by prolonging the PC patients' survival time, which benefit from reconstructed immune function of patients.

Keywords: cytokine-induced killer cells, dendritic cells, pancreatic cancer, immunotherapy, meta-analysis

\section{Introduction}

Pancreatic cancer (PC) is a fatal disease with high mortality and poor prognosis. ${ }^{1}$ It is the twelfth most common cancer and is the seventh leading cause of cancer-related deaths in the world with 338,000 new cases per year. ${ }^{2}$ In recent years, PC incidence has been significantly raised. The median overall survival (OS) of patients with advanced $\mathrm{PC}$ is $4-6$ months, ${ }^{3}$ and the 5 -year OS rate is $<10 \% .{ }^{4}$ Common therapeutic options for PC are surgery, radiotherapy, and chemotherapy, ${ }^{3}$ but none of these strategies were able to thoroughly remove small residuals and metastatic cells, which is a main problem to be solved in tumor therapeutics. Therefore, effective therapeutic method should be developed. 
Adoptive cellular immunotherapy has demonstrated efficacy for the treatment of various malignant tumors, especially dendritic cells-cytokine-induced killer (DC-CIK) cells mediate immunotherapy. ${ }^{5,6}$ Compared to immunotherapy using other types of cells, such as lymphokine-activated killer (LAK) cells, ${ }^{7,8}$ natural killer (NK) cells, ${ }^{9,10}$ and other immune cells, ${ }^{11,12}$ DC-CIK-mediated immunotherapy exhibited a stronger antitumor ability and broader antitumor spectrum. ${ }^{13,14}$ Cytokine-induced killer (CIK) cell is a heterogeneous subset of $\mathrm{T}$ lymphocytes, which primarily consist of $\mathrm{CD} 3{ }^{+} \mathrm{CD} 56^{+}$ cells and is easy to be collected from human peripheral and umbilical cord blood, and subsequently induced by IFN- $\gamma$, anti-CD3 monoclonal antibodies (OKT-3), and IL-2 in vitro. ${ }^{6}$ $\mathrm{DC}$ are the most potent antigen-presenting cells. DC have the capacity to enhance CIK's cytotoxicity by coculture with CIK cells, which is indicated by increased proportion of $\mathrm{CD}^{+}{ }^{+} \mathrm{CD} 56^{+}$cells and improved levels of cytokines such as IFN- $\gamma$ and IL-2., ${ }^{5}$

Clinical application of DC-CIK immunotherapy for PC has been reported in several clinical trials. ${ }^{16-19}$ In a metaanalysis comparing cellular immunotherapy combined with chemotherapy and chemotherapy alone, the former showed significantly prolonged $\mathrm{OS},{ }^{3}$ while the discussed outcomes were not complete. Analysis considering overall response rate (ORR) and disease control rate (DCR), patients' quality of life (QoL) and safety were not involved in this analysis. Moreover, the immunotherapy regimens among studies were different (including DC, NK, and LAK), which may influence the analysis of clinical therapy. Our study focused on PC patients treated by DC-CIK immunotherapy and chemotherapy combined therapy or chemotherapy alone, and we performed an up-to-date meta-analysis to provide reliable evidence on the efficacy and safety of DC-CIK immunotherapy in treating PC patients.

\section{Methods}

\section{Search strategy and selection criteria}

Data were collected from Web of Science, EMBASE, PubMed, Cochrane Library, Wanfang, and CNKI databases using the key terms of "dendritic cells", "cytokine-induced killer cells" combined with "pancreatic cancer". No language limits were applied. Literature published before May 2017 was involved in our analysis.

The main selection criteria are that $\mathrm{PC}$ patients in the experimental group underwent DC-CIK immunotherapy combined with chemotherapy and patients in the control group were treated by chemotherapy alone.

\section{Data collection and quality assessment}

Literature screening and data extraction were carried out by two independent reviewers (YZ and XZ), and disagreements were eliminated upon discussing with a third researcher (AZ). Extracted information included first author's names, years of publication, study locations, tumor stages, number of cases, patient ages, therapeutic regimens, administration route, in vitro cell culture conditions, and dosages of utilized immune cells. The quality of the included trials was evaluated based on Cochrane Handbook. ${ }^{20}$

\section{Treatment efficacy}

Treatment efficacy was assessed in terms of the complete response (CR) rates, partial response (PR) rates, stable disease $(\mathrm{SD})$ rates, progressive disease $(\mathrm{PD})$ rates, ORR, $(\mathrm{ORR}=\mathrm{CR}+\mathrm{PR})$ and $\mathrm{DCR}(\mathrm{DCR}=\mathrm{CR}+\mathrm{PR}+\mathrm{SD})$. Prognosis was estimated by OS, which was defined as the length of time from the start of treatment to the death of patient from any cause, ${ }^{21}$ patients' QoL, and adverse events. Immune function of PC patients before and after treatment was determined by lymphocyte subsets' percentages ( $\mathrm{CD} 3^{+}$, $\mathrm{CD}^{+}, \mathrm{CD}^{+}, \mathrm{CD}^{+} \mathrm{CD}^{2} 6^{+}$, and $\left.\mathrm{CD} 4^{+} \mathrm{CD} 25^{+} \mathrm{CD} 127^{\text {low }}\right)$ and cytokines secretion levels (IFN- $\gamma$ and IL-4).

\section{Statistical analysis}

This meta-analysis was performed using RevMan 5.2 (version 5.2, Nordic Cochran Centre, Copenhagen, Denmark). $P<0.05$ indicates the statistical significance of the difference. Heterogeneity among studies was assessed to determine suitable analysis model. ${ }^{5,22}$ Cochran's $Q$ test was performed to evaluate the homogeneity, and funnel plots were used to assess the publication bias of included studies. $I^{2}<50 \%$ or $P>0.1$ indicated that the studies were homogenous. Odds ratio (OR) was the principal measurement for treatment efficacy and is presented with a $95 \%$ confidence interval (CI). Sensitivity analysis was conducted to evaluate the consistency of the results and evaluate the influence of single studies on overall risk estimate. ${ }^{23}$

\section{Results}

\section{Search results}

A total of 2,127 articles were identified upon initial retrieve; 2,082 articles were excluded because they lacked clinical trial $(n=1,876)$, were unrelated studies $(n=64)$, and were duplicated $(n=142)$. After a detailed assessment of full texts, 14 reviews or meta-analyses, 10 articles without control 


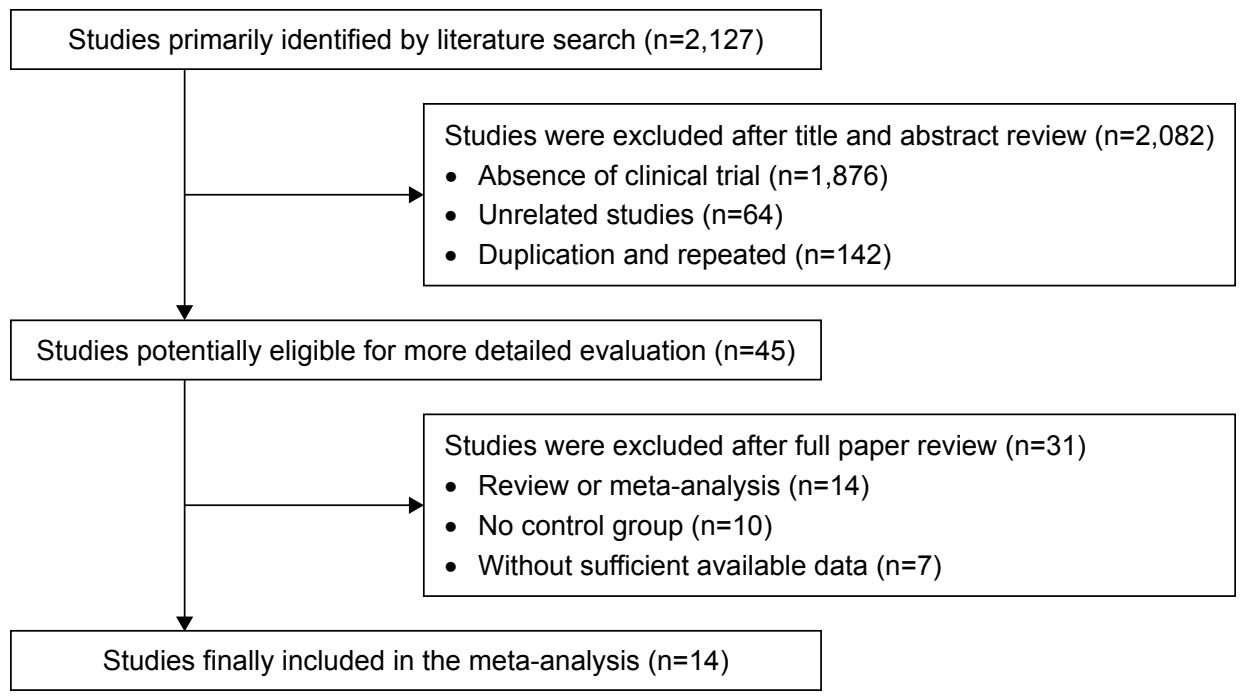

Figure I Flow diagram of the selection process.

group, and seven papers with insufficient data were excluded. Finally, 14 papers of clinical trials that included a total of 1,088 patients were eligible for inclusion in this meta-analysis (Figure 1). ${ }^{16,17,24-35}$

\section{Patient's characteristics}

All of the involved trials turn out to be conducted in China. In total, 513 PC patients in eight trials were treated by DCCIK in combination with chemotherapy, while 575 patients in six trials were treatment by CIK alone. Detailed clinical information of the patients is presented in Table 1. DC and CIK cells were obtained from autologous peripheral blood, confirming free of bacterial and fungal contaminations before venous transfusion to the patients. Information of DC-CIK mediate immunotherapy is shown in Table 2.

\section{Quality assessment}

Bias risk assessment is shown in Figure 2. Seven studies were determined as low risk, four researches were not truly randomized controlled trials, and the remaining three studies lacked clear description of randomization process. Risks of allocation, performance, and detection were low. Two studies absent of follow-up, seven trials with selective

Table I Clinical information from the eligible trials in the meta-analysis

\begin{tabular}{|c|c|c|c|c|c|c|}
\hline \multirow[t]{2}{*}{ Included studies } & \multirow[t]{2}{*}{ Nation } & \multirow{2}{*}{$\begin{array}{l}\text { Tumor } \\
\text { stage }\end{array}$} & \multirow{2}{*}{$\begin{array}{l}\text { Patients, } \\
\text { exp/con }\end{array}$} & \multicolumn{2}{|l|}{ Age (years) } & \multirow[t]{2}{*}{ Parameter types } \\
\hline & & & & Exp & Con & \\
\hline Ge and $\mathrm{Ge}(2016)^{24}$ & China & I-IV & $50 / 50$ & $57.7 \pm 4.6$ (mean) & $57.5 \pm 4.7$ (mean) & OS, LYM subsets \\
\hline Kang and Zhang $(2016)^{25}$ & China & Kps $>60$ & $22 / 22$ & $65.1 \pm 6.3$ (mean) & $66.1 \pm 6.3$ (mean) & ORR, DCR, QoL \\
\hline $\operatorname{Li}(2016)^{26}$ & China & Kps $\geq 70$ & $27 / 27$ & ND & ND & OS, ORR, DCR, QoL, AE \\
\hline Liu $(2012)^{27}$ & China & I-IV & $25 / 25$ & ND & ND & LYM subsets, cytokines \\
\hline Mu et al $(2016)^{28}$ & China & III-IV & $90 / 90$ & $56.5 \pm 8.3$ (mean) & $57.8 \pm 7.3$ (mean) & ORR, DCR, LYM subsets, AE \\
\hline Shen et al $(2015)^{29}$ & China & III-IV & $38 / 36$ & 62 (median) & 66 (median) & ORR, DCR \\
\hline Wang $(2015)^{30}$ & China & I-IV & $10 / 30$ & $64.3 \pm 3.1$ (mean) & $63.8 \pm 3.4$ (mean) & ORR, DCR \\
\hline Wang et al $(2013)^{16}$ & China & $P s \leq 2$ & $28 / 30$ & ND & ND & ORR, DCR, AE \\
\hline Wang et al $(2016)^{17}$ & China & $\mathrm{Ps}<3$ & $25 / 5$ & $<65(14)$ & $<65(38)$ & OS, ORR, DCR, AE \\
\hline Wen et al $(2013)^{31}$ & China & ND & $30 / 30$ & $63.5 \pm 13.2$ (mean) & $65.3 \pm 12.8$ (mean) & OS, ORR, DCR \\
\hline Zhang et al $(2013)^{32}$ & China & Kps $>60$ & $58 / 68$ & 63 (median) & 65 (median) & ORR, DCR, QoL \\
\hline Zhang $(2014)^{33}$ & China & I-IV & $30 / 30$ & ND & ND & OS, LYM subsets, cytokines \\
\hline Zhang et al $(2016)^{34}$ & China & ND & $40 / 40$ & $55.9 \pm 8.7$ (mean) & $56.8 \pm 8.2$ (mean) & OS \\
\hline Zheng et al $(2016)^{35}$ & China & I-IV & $40 / 40$ & $39-82$ & $35-83$ & OS, LYM subsets \\
\hline
\end{tabular}

Notes: Con, control group (chemotherapy alone group); Exp, experimental group (chemotherapy with DC-CIK immunotherapy).

Abbreviations: AE, adverse reaction; DC-CIK, dendritic cells-cytokine-induced killer; DCR, disease control rate; Kps, Karnofsky Performance Score; LYM, lymphocyte; ND, not determined; OS, overall survival; ORR, overall response rate; Ps, performance status score; QoL, quality of life. 
Table 2 Information of DC-CIK immunotherapy

\begin{tabular}{|c|c|c|c|c|c|}
\hline \multirow[t]{2}{*}{ Included studies } & \multicolumn{2}{|l|}{ Therapeutic regimen } & \multirow{2}{*}{$\begin{array}{l}\text { Administration } \\
\text { route }\end{array}$} & \multirow{2}{*}{$\begin{array}{l}\text { Culture } \\
\text { conditions }\end{array}$} & \multirow[t]{2}{*}{ Cell dose (cycles) } \\
\hline & Experimental group & Control group & & & \\
\hline $\mathrm{Ge}$ and $\mathrm{Ge}(20 \mid 6)^{24}$ & Con Reg + DC-CIK & Gemcitabine + oxaliplatin +5 -Fu & Intravenous infusion & ND & $6 \times 10^{9}$ ( 2 cycles $)$ \\
\hline Kang and Zhang $(2016)^{25}$ & Con Reg + DC-CIK & Gemcitabine & ND & ND & ND \\
\hline $\mathrm{Li}(2016)^{26}$ & Con Reg + CIK & Gemcitabine + cisplatin + RT & Intravenous infusion & ND & $>1 \times 10^{10}(\geq 2$ cycles $)$ \\
\hline Liu $(20 \mid 2)^{27}$ & Con Reg + DC-CIK & ND & Intravenous infusion & $\begin{array}{l}\text { IFN- } \gamma \text {, IL-2, CD3, } \\
\text { GM-CSF, IL-4 }\end{array}$ & $6 \times 10^{9}$ ( 2 cycles $)$ \\
\hline Mu et al $(2016)^{28}$ & Con Reg + DC-CIK & Gemcitabine + nab-paclitaxel & Intravenous infusion & ND & ND (4 cycles) \\
\hline Shen et al $(2015)^{29}$ & Con Reg + CIK & Gemcitabine & Intravenous infusion & IFN- $\gamma$, IL-2, CD3 & ND \\
\hline Wang $(2015)^{30}$ & Con Reg + CIK & Gemcitabine & Intravenous infusion & ND & ND ( 2 cycles $)$ \\
\hline Wang et al $(2013)^{16}$ & Con Reg + CIK & S-I & Intravenous infusion & $\begin{array}{l}\text { IFN- } \gamma \text {, IL-I } \alpha \text {, } \\
\text { IL-2, CD3 }\end{array}$ & ND ( $\geq$ I cycle) \\
\hline Wang et al $(2016)^{17}$ & Con Reg + CIK & (gemcitabine + S-I)/other & Intravenous infusion & IFN- $\gamma$, IL-2, CD3 & $5 \times 10^{9}$ ( 2 cycles $)$ \\
\hline Wen et al $(2013)^{31}$ & Con Reg + DC-CIK & Mitomycin + adriamycin + Fu & Intravenous infusion & ND & ND \\
\hline Zhang et al $(2013)^{32}$ & Con Reg + CIK & Gemcitabine & Intravenous infusion & ND & ND \\
\hline Zhang $(2014)^{33}$ & Con Reg + DC-CIK & Gemcitabine + oxaliplatin +5 -Fu & Intravenous infusion & GM-CSF, IL-4 & ND \\
\hline Zhang et al $(2016)^{34}$ & Con Reg + DC-CIK & Gemcitabine + oxaliplatin +5 -Fu & Intravenous infusion & ND & $>1 \times 10^{9}(\mathrm{ND})$ \\
\hline Zheng et al $(2016)^{35}$ & Con Reg + DC-CIK & Gemcitabine + oxaliplatin +5 -Fu & Intravenous infusion & ND & $6 \times 10^{9}$ ( 2 cycles $)$ \\
\hline
\end{tabular}

Notes: Con, control group (chemotherapy alone group); exp, experimental group (chemotherapy with DC-CIK immunotherapy); RT, three-dimensional conformal radiotherapy.

Abbreviations: Con Reg, control group regimen; DC-CIK, dendritic cells-cytokine-induced killer; ND, not determined; 5-Fu, 5-fluorouracil; GM-CSF, granulocytemacrophage colony-stimulating factor.

reporting were regarded as an unclear risk, and other two studies were considered as high risk for lacking primary outcome data.

\section{Efficacy assessments}

In our pooled analysis, patients treated by combined therapy showed higher $\mathrm{PR}$ (Figure $\mathrm{S} 1 \mathrm{~A}, \mathrm{OR}=1.49,95 \% \mathrm{CI}=1.06-2.10$, $P=0.02$ ), ORR (Figure 3A, OR $=1.69,95 \% \mathrm{CI}=1.20$ 2.38, $P=0.003$ ), and DCR (Figure 3B, OR $=2.33,95 \%$ $\mathrm{CI}=1.63-3.33, P<0.00001)$ and lower $\mathrm{PD}$ rates (Figure S1B,
$\mathrm{OR}=0.43,95 \% \mathrm{CI}=0.30-0.61, P<0.00001)$ with statistical significance, whereas $\mathrm{CR}$ and $\mathrm{SD}$ did not show obvious difference from chemo-alone group (Figure S1C and D, CR: $\mathrm{OR}=1.97,95 \% \mathrm{CI}=0.85-4.54, P=0.11 ; \mathrm{SD}: \mathrm{OR}=1.31,95 \%$ $\mathrm{CI}=0.95-1.80, P=0.10)$. Fixed-effect models were used in this analysis because of low heterogeneity (Table 3).

\section{Prognosis evaluation}

In the 14 studies, patients treated by combined therapy had higher OS than those treated by chemotherapy alone (Figure 4,

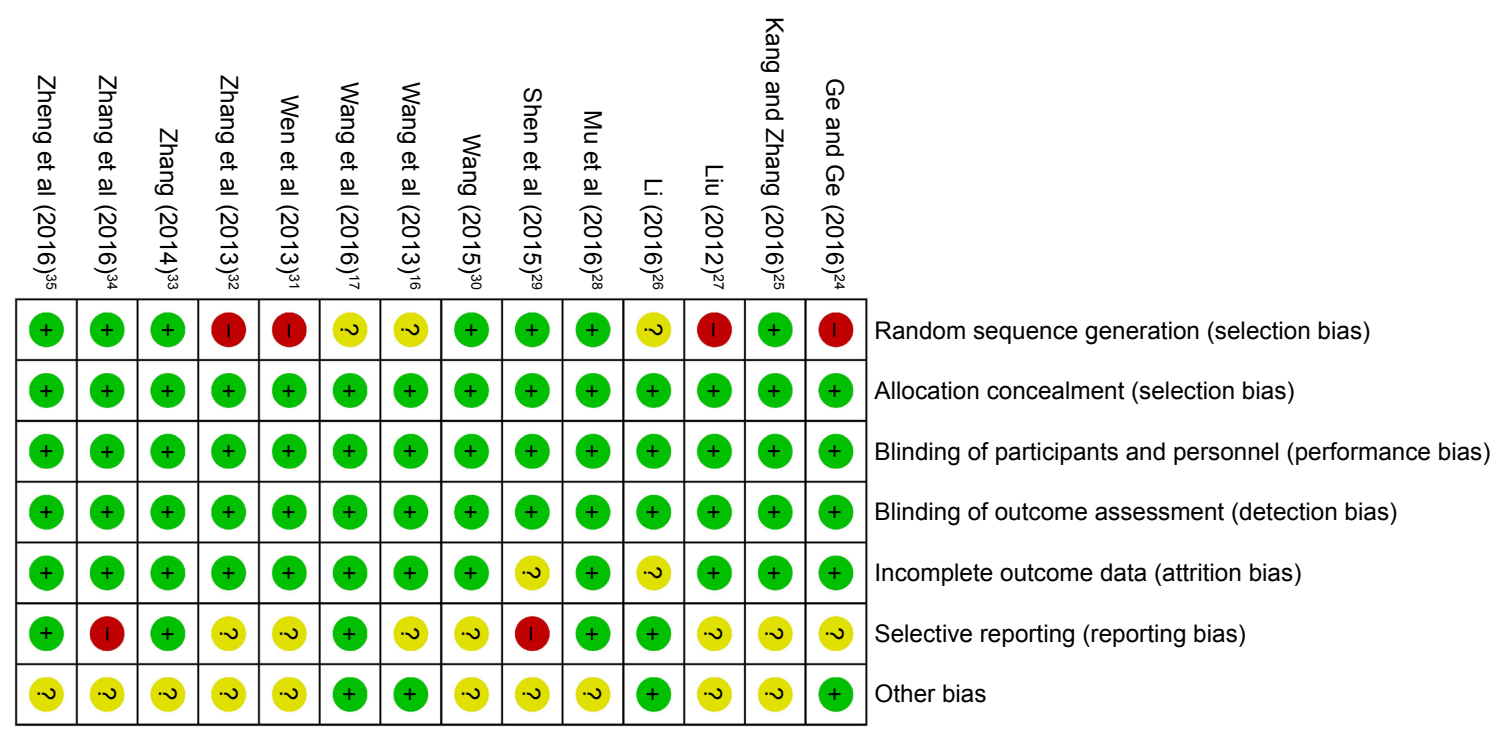

Figure 2 Risk of bias summary: review of authors' judgments about each risk of bias item for included studies.

Note: Each color represents a different level of bias: red for high-risk, green for low-risk, and yellow for unclear-risk of bias. 


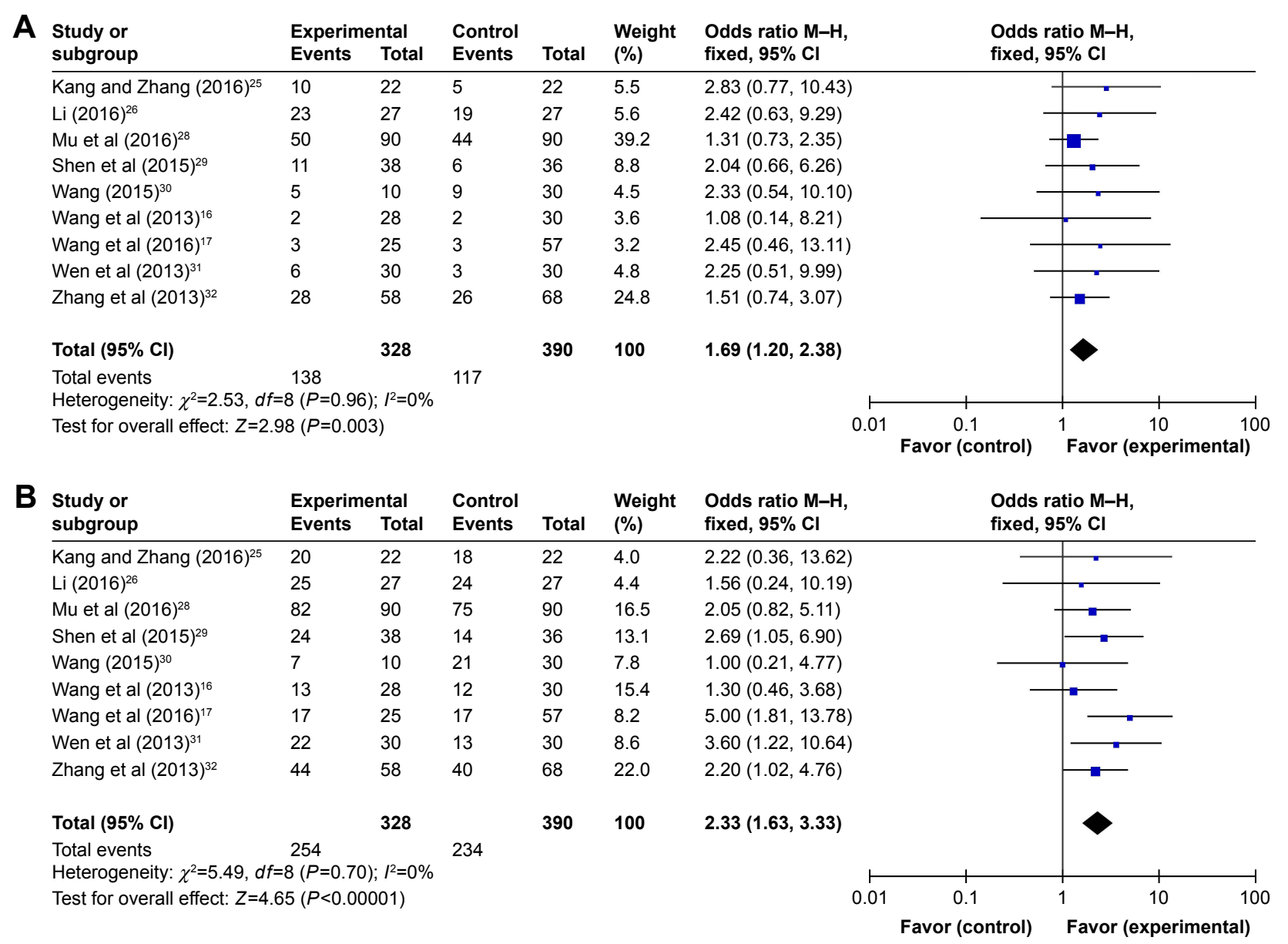

Figure 3 Forest plots of the comparison of ORR (A) and DCR (B) between the experimental and control groups.

Notes: Control group, chemotherapy alone group; experimental group, chemotherapy with DC-CIK immunotherapy. The fixed-effects meta-analysis model (M-H method) was used.

Abbreviations: $\mathrm{Cl}$, confidence interval; DC-CIK, dendritic cells-cytokine-induced killer; DCR, disease control rate; $\mathrm{M}-\mathrm{H}$, Mantel-Haenszel; ORR, overall response rate.

1-year OS: $\mathrm{OR}=3.61,95 \% \mathrm{CI}=2.41-5.40, P<0.00001 ; 3$-year OS: $\mathrm{OR}=2.65,95 \% \mathrm{CI}=1.56-4.50, P=0.0003)$. Considering slightly significant heterogeneity, fixed-effects model were applied in this analysis. Compared to control group, patients in experimental group showed significantly improved QoL (Figure 5A, OR =3.04, CI $=1.58-5.88, P=0.0009$ ) and Karnofsky Performance Score (Kps) (Figure 5B, OR =9.06,
95\% CI $=7.27-10.84, P<0.00001)$, which also indicates the performance status of patients.

\section{Immune function evaluation}

The immune status of patients was examined before and after treatment. As shown in Figure 6, after DC-CIK treatment, percentages of $\mathrm{CD}^{+}, \mathrm{CD}^{+}$, and $\mathrm{CD}^{+} \mathrm{CD}^{+} 6^{+}$

Table 3 Comparison of CR, PR, SD, PD, ORR, and DCR between the experimental and control groups

\begin{tabular}{|c|c|c|c|c|c|c|c|c|}
\hline \multirow[t]{2}{*}{ Parameter } & \multicolumn{2}{|c|}{ Number of patients $(n)$} & \multirow{2}{*}{$\begin{array}{l}\text { Analysis } \\
\text { method }\end{array}$} & \multicolumn{2}{|c|}{ Heterogeneity } & \multirow[t]{2}{*}{ OR } & \multirow[t]{2}{*}{$95 \% \mathrm{Cl}$} & \multirow[t]{2}{*}{$P$-value } \\
\hline & $\begin{array}{l}\text { Experimental } \\
\text { group }\end{array}$ & $\begin{array}{l}\text { Control } \\
\text { group }\end{array}$ & & $I^{2}(\%)$ & $P$-value & & & \\
\hline $\mathrm{CR}$ & 328 & 390 & Fixed & 0 & 0.84 & 1.97 & $0.85-4.54$ & 0.11 \\
\hline PR & 328 & 390 & Fixed & 0 & 0.96 & 1.49 & $1.06-2.10$ & 0.02 \\
\hline SD & 328 & 390 & Fixed & 35 & 0.14 & 1.31 & $0.95-1.80$ & 0.10 \\
\hline PD & 328 & 390 & Fixed & 0 & 0.74 & 0.43 & $0.30-0.6 \mathrm{I}$ & $<0.0000$ I \\
\hline ORR & 328 & 390 & Fixed & 0 & 0.96 & 1.69 & I.20-2.38 & 0.003 \\
\hline DCR & 328 & 390 & Fixed & 0 & 0.70 & 2.33 & $1.63-3.33$ & $<0.00001$ \\
\hline
\end{tabular}

Abbreviations: $\mathrm{Cl}$, confidence interval; $\mathrm{CR}$, complete response; $\mathrm{DCR}$, disease control rate; OR, odds ratio; ORR, overall response rate; PR, partial response; $\mathrm{PD}$, progressive disease; SD, stable disease. 


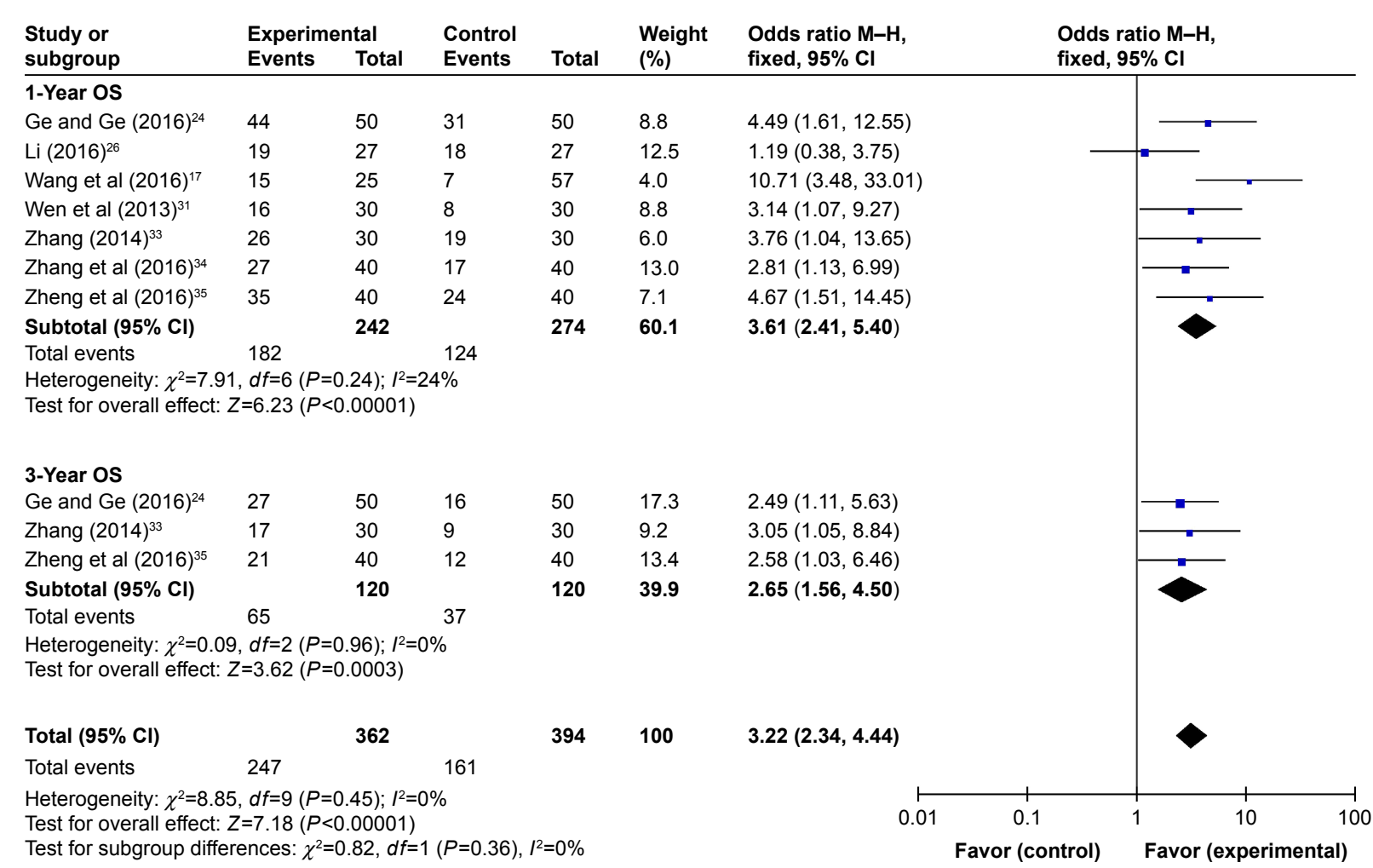

Figure 4 Forest plot of the comparison of OS between the experimental and control groups.

Notes: Control group, chemotherapy alone group; experimental group, chemotherapy with DC-CIK immunotherapy. The fixed-effects meta-analysis model (M-H method) was used.

Abbreviations: $\mathrm{Cl}$, confidence interval; DC-CIK, dendritic cells-cytokine-induced killer; $\mathrm{M}-\mathrm{H}$, Mantel-Haenszel; OS, overall survival.

were increased $\left(\mathrm{CD}^{+}\right.$: $\mathrm{OR}=10.70,95 \% \mathrm{CI}=7.38-14.03$, $P<0.00001 ; \mathrm{CD}^{+}$: $\mathrm{OR}=7.62,95 \% \mathrm{CI}=1.56-13.67, P=0.01$; $\left.\mathrm{CD}^{+}{ }^{+} \mathrm{CD} 56^{+}: \mathrm{OR}=7.34,95 \% \mathrm{CI}=6.77-7.92, P<0.00001\right)$, and percentage of $\mathrm{CD} 4^{+} \mathrm{CD} 25^{+} \mathrm{CD} 127^{\text {low }}$ was decreased
( $\mathrm{OR}=-3.52,95 \% \mathrm{CI}=-4.61$ to $-2.44, P<0.00001$ ); the changes were statistically significant, whereas proportions of $\mathrm{CD}^{+}$and $\mathrm{CD}^{+} / \mathrm{CD}^{+}{ }^{+}$ratio were not apparently changed $\left(\mathrm{CD}^{+}\right.$: $\mathrm{OR}=-5.01,95 \% \mathrm{CI}=-23.14$ to $13.12, P=0.59 ; \mathrm{CD}^{+} / \mathrm{CD}^{+}$

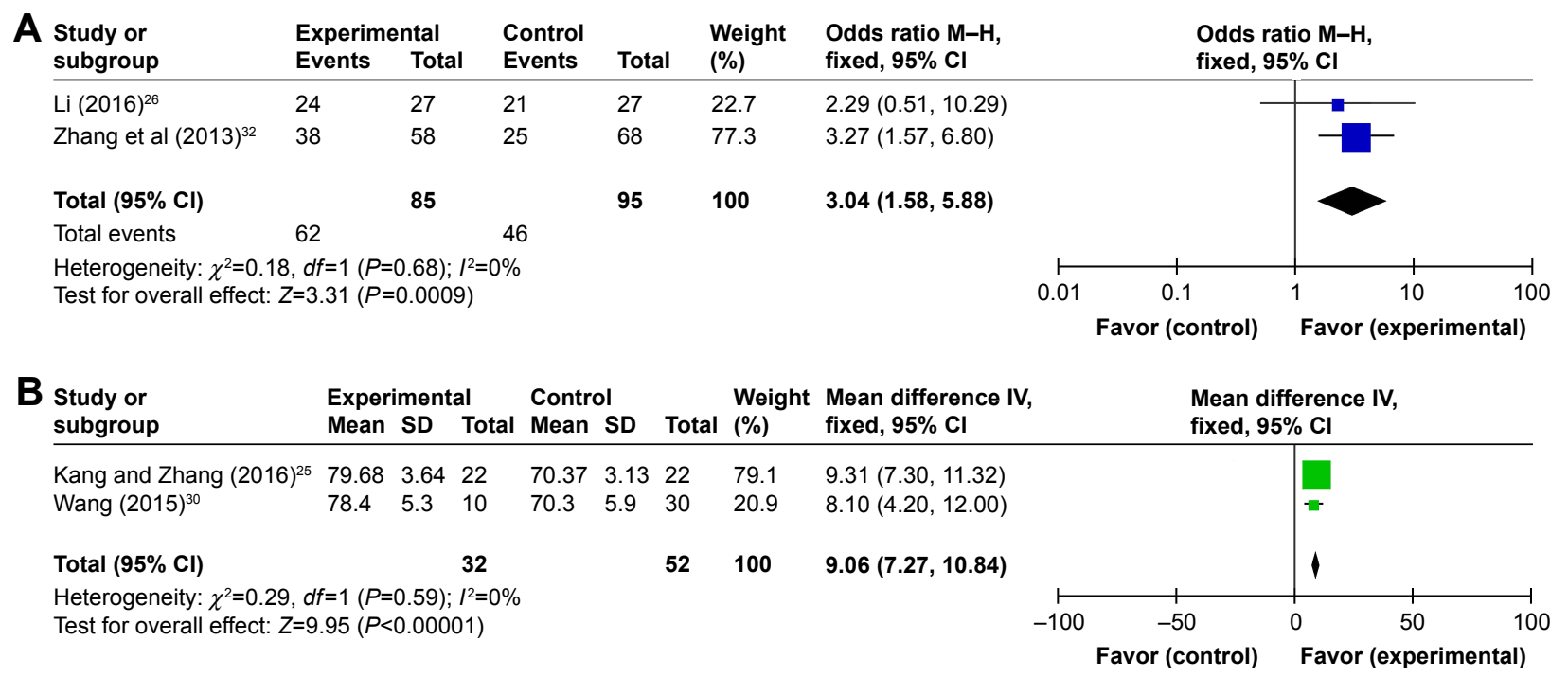

Figure 5 Forest plots of the comparison of QoL between the experimental and control groups.

Notes: (A) QoL improvement; (B) Kps. Control group, chemotherapy alone group; experimental group, chemotherapy with DC-CIK immunotherapy. The fixed-effects meta-analysis model was used.

Abbreviations: Cl, confidence interval; DC-CIK, dendritic cells-cytokine-induced killer; Kps, Karnofsky Performance Score; M-H, Mantel-Haenszel; QoL, quality of life. 


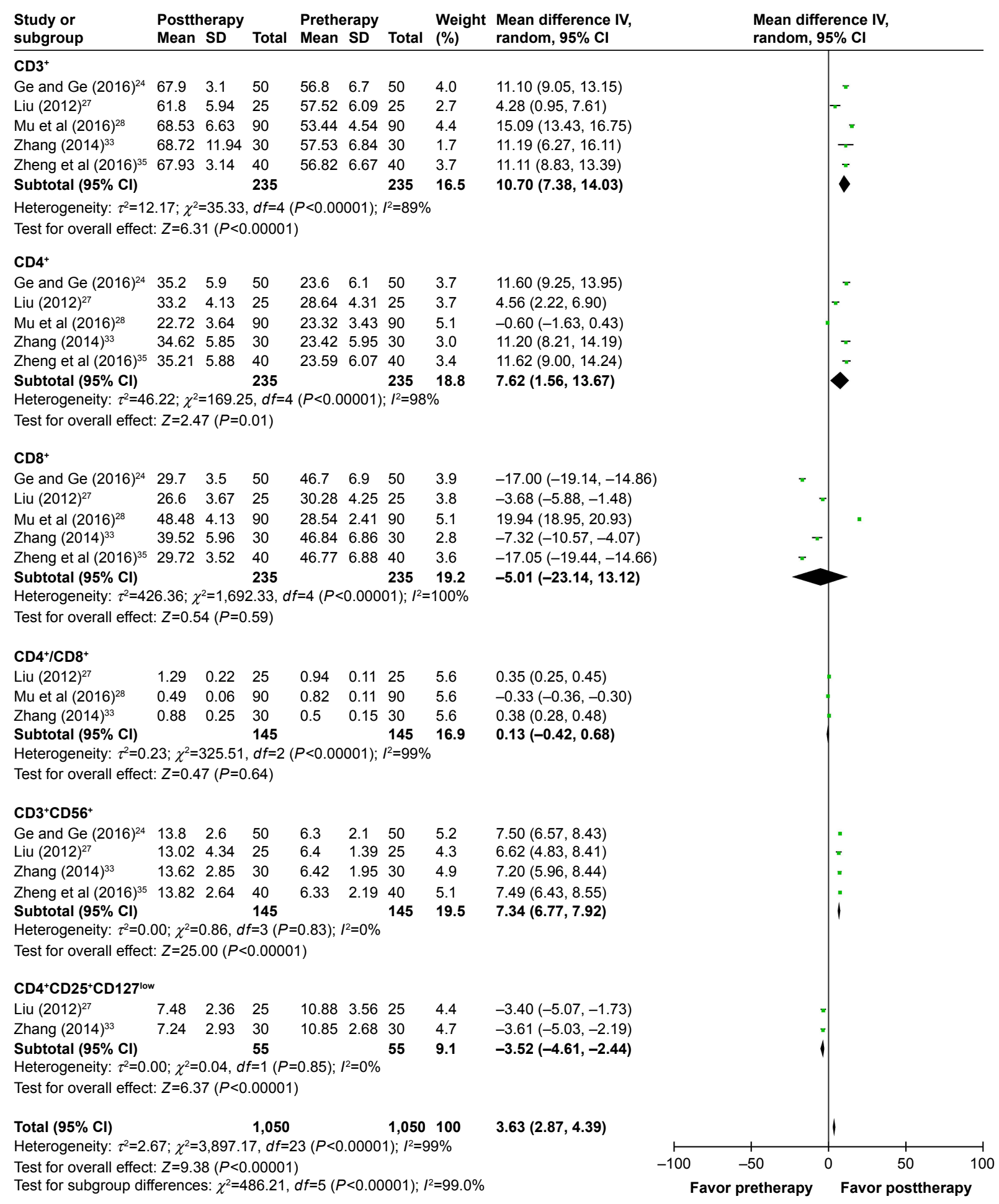

Figure 6 Forest plot of the comparison of immunophenotype in pre- and posttherapies. Note: The random effects meta-analysis model (IV method) was used.

Abbreviations: $\mathrm{Cl}$, confidence interval; IV, inverse variance.

ratio: $\mathrm{OR}=0.13,95 \% \mathrm{CI}=-0.42$ to $0.68, P=0.64)$. In contrast, IFN- $\gamma$ level was increased distinctly after DC-CIK immunotherapy (IFN- $\gamma$ : $\mathrm{OR}=2.28,95 \% \mathrm{CI}=1.33$ to $3.22, P<0.00001$ ), while IL-4 level was dramatically decreased $(\mathrm{OR}=-1.85,95 \%$ $\mathrm{CI}=-2.69$ to $-1.01, P<0.0001$ ) (Figure 7 ).

\section{Adverse events' assessment}

In the involved clinical trials, no serious adverse events or death occurrence was reported in patients receiving DCCIK immunotherapy. As shown in Figures S2 and 3 and Table 4, no significant difference was found on adverse 


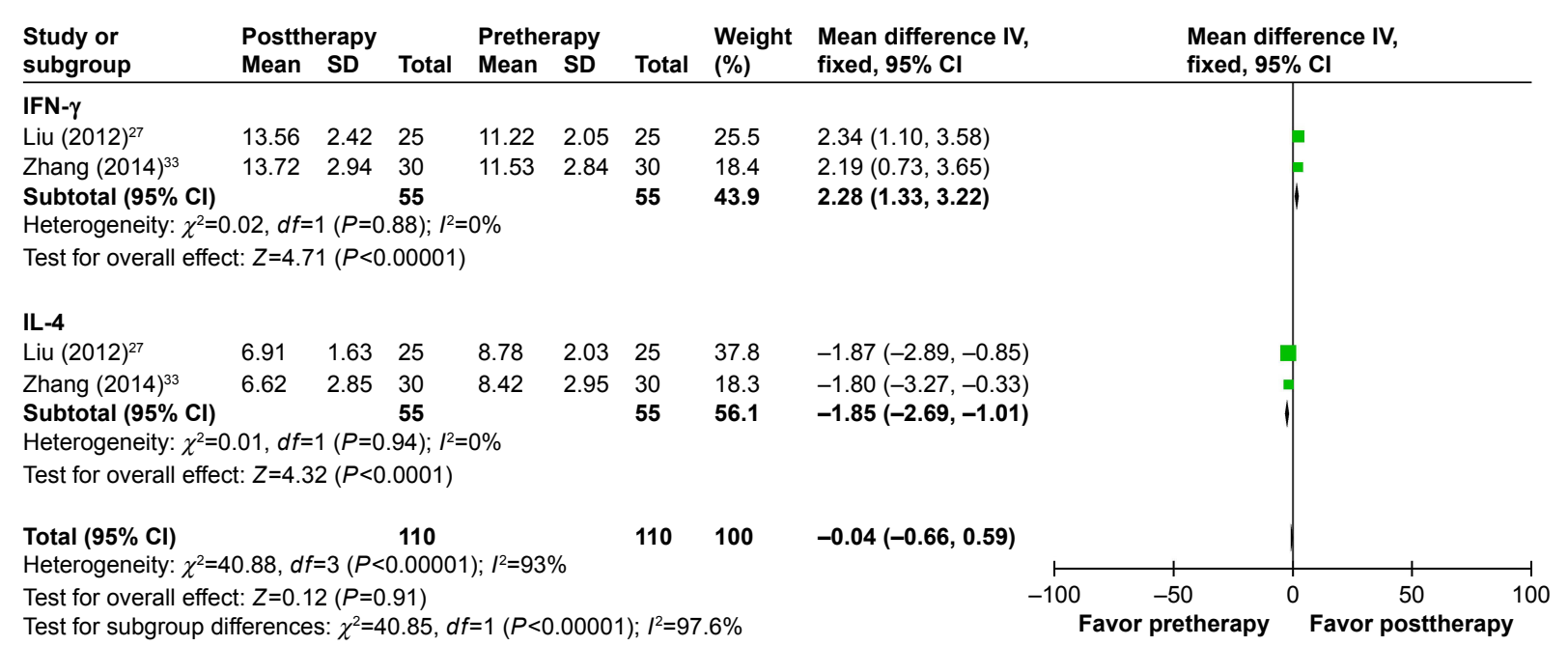

Figure 7 Forest plot of the comparison of IFN- $\gamma$ and IL-4 in pre- and posttherapies.

Note: The fixed-effects meta-analysis model (IV method) was used.

Abbreviations: $\mathrm{Cl}$, confidence interval; IV, inverse variance; IFN- $\gamma$, interferon- $\gamma$; IL-4, interleukin-4.

events including fever, skin rash, leukopenia, thrombocytopenia, diarrhea, nausea and vomiting, gastrointestinal adverse reaction (AE), fatigue, neutropenia, and myelosuppression between the experimental and control groups (fever: $\mathrm{OR}=2.39,95 \% \mathrm{CI}=0.70-8.23, P=0.17$ [Figure $\mathrm{S} 3 \mathrm{~A}$, fever I + II: OR $=4.34,95 \% \mathrm{CI}=1.35-13.89, P=0.01$; fever III + IV: OR $=3.11,95 \% \mathrm{CI}=0.12-79.87, P=0.49]$; skin rash: $\mathrm{OR}=1.32,95 \% \mathrm{CI}=0.54-3.19, P=0.54$ [Figure S3B, skin rash I + II: OR $=2.21,95 \% \mathrm{CI}=0.52-9.36, P=0.28$; skin rash III + IV: OR $=3.33,95 \% \mathrm{CI}=0.13-85.11$, $P=0.47]$; leukopenia: $\mathrm{OR}=0.56,95 \% \mathrm{CI}=0.22-1.47$, $P=0.24$ [Figure S3C, leukopenia I + II: OR $=0.86,95 \%$ $\mathrm{CI}=0.36-2.06, P=0.73$; leukopenia III $+\mathrm{IV}: \mathrm{OR}=0.32,95 \%$ $\mathrm{CI}=0.06-1.64, P=0.17]$; thrombocytopenia: $\mathrm{OR}=0.54,95 \%$

Table 4 Comparison of adverse events between the experimental and control groups

\begin{tabular}{|c|c|c|c|c|c|c|c|c|}
\hline \multirow[t]{2}{*}{ Adverse events } & \multicolumn{2}{|c|}{ Number of patients (n) } & \multirow{2}{*}{$\begin{array}{l}\text { Analysis } \\
\text { method }\end{array}$} & \multicolumn{2}{|c|}{ Heterogeneity } & \multirow[t]{2}{*}{ OR } & \multirow[t]{2}{*}{$95 \% \mathrm{Cl}$} & \multirow[t]{2}{*}{$P$-value } \\
\hline & $\begin{array}{l}\text { Experimental } \\
\text { group }\end{array}$ & $\begin{array}{l}\text { Control } \\
\text { group }\end{array}$ & & $I^{2}(\%)$ & $P$-value & & & \\
\hline Fever & 170 & 204 & Random & 67 & 0.03 & 2.39 & $0.70-8.23$ & 0.17 \\
\hline Fever I + II & 55 & 57 & Random & 57 & 0.13 & 4.34 & $1.35-13.89$ & 0.01 \\
\hline Fever III + IV & 55 & 57 & Random & & & 3.11 & $0.12-79.87$ & 0.49 \\
\hline Skin rash & 145 & 147 & Random & 0 & 0.46 & 1.32 & $0.54-3.19$ & 0.54 \\
\hline Skin rash I + II & 55 & 57 & Random & 0 & 0.66 & 2.21 & $0.52-9.36$ & 0.28 \\
\hline Skin rash III + IV & 55 & 57 & Random & & & 3.33 & $0.13-85.11$ & 0.47 \\
\hline Leukopenia & 55 & 57 & Random & 0 & 0.33 & 0.56 & $0.22-1.47$ & 0.24 \\
\hline Leukopenia I + II & 55 & 57 & Random & 0 & 0.92 & 0.86 & $0.36-2.06$ & 0.73 \\
\hline Leukopenia III + IV & 55 & 57 & Random & 0 & 0.62 & 0.32 & $0.06-1.64$ & 0.17 \\
\hline Thrombocytopenia & 55 & 57 & Random & 0 & 0.38 & 0.54 & $0.18-1.64$ & 0.27 \\
\hline Thrombocytopenia I + II & 55 & 57 & Random & 0 & 0.70 & 0.61 & $0.22-1.73$ & 0.36 \\
\hline Thrombocytopenia III + IV & 55 & 57 & Random & & & 0.32 & $0.01-8.24$ & 0.49 \\
\hline Diarrhea & 55 & 57 & Random & 0 & 0.48 & 1.52 & $0.57-4.03$ & 0.40 \\
\hline Diarrhea I + II & 55 & 57 & Random & 0 & 0.49 & 1.58 & $0.58-4.32$ & 0.37 \\
\hline Diarrhea III + IV & 55 & 57 & Random & & & 1.08 & $0.14-8.21$ & 0.94 \\
\hline Nausea, vomiting & 55 & 57 & Random & 0 & 0.42 & 0.83 & $0.30-2.28$ & 0.72 \\
\hline Nausea, vomiting I + II & 55 & 57 & Random & 0 & 0.49 & 0.95 & $0.35-2.60$ & 0.92 \\
\hline Nausea, vomiting III + IV & 55 & 57 & Random & & & 0.35 & $0.0 \mathrm{I}-8.83$ & 0.52 \\
\hline Gastrointestinal AE & 52 & 84 & Random & 0 & 0.32 & 0.65 & $0.23-1.90$ & 0.43 \\
\hline Fatigue & 53 & 87 & Random & 72 & 0.06 & 0.66 & $0.08-5.80$ & 0.71 \\
\hline Neutropenia & 28 & 30 & Random & & & 1.09 & $0.28-4.25$ & 0.90 \\
\hline Myelosuppression & 25 & 57 & Random & & & 0.48 & $0.19-1.26$ & 0.14 \\
\hline
\end{tabular}

Abbreviations: $\mathrm{AE}$, adverse reaction; $\mathrm{Cl}$, confidence interval; $\mathrm{OR}$, odds ratio. 
$\mathrm{CI}=0.18-1.64, P=0.27$ [Figure $\mathrm{S} 3 \mathrm{D}$, thrombocytopenia I + II: $\mathrm{OR}=0.61,95 \% \mathrm{CI}=0.22-1.73, P=0.36$; thrombocytopenia III + IV: OR $=0.32,95 \% \mathrm{CI}=0.01-8.24, P=0.49]$; diarrhea: $\mathrm{OR}=1.52,95 \% \mathrm{CI}=0.57-4.03, P=0.40$ [Figure S3E, diarrhea I + II: $\mathrm{OR}=1.58,95 \% \mathrm{CI}=0.58-4.32, P=0.37$; diarrhea III + IV: OR $=1.08,95 \% \mathrm{CI}=0.14-8.21, P=0.94]$; nausea and vomiting: $\mathrm{OR}=0.83,95 \% \mathrm{CI}=0.30-2.28$, $P=0.72$ [Figure S3F, nausea and vomiting I + II: $\mathrm{OR}=0.95$, $95 \% \mathrm{CI}=0.35-2.60, P=0.92$; nausea and vomiting III $+\mathrm{IV}$ : $\mathrm{OR}=0.35,95 \% \mathrm{CI}=0.01-8.83, P=0.52]$; gastrointestinal $\mathrm{AE}$ : $\mathrm{OR}=0.65,95 \% \mathrm{CI}=0.23-1.90, P=0.43$; fatigue: $\mathrm{OR}=0.66$, 95\% CI $=0.08-5.80, P=0.71$; neutropenia: $\mathrm{OR}=1.09,95 \%$ $\mathrm{CI}=0.28-4.25, P=0.90$; and myelosuppression: $\mathrm{OR}=0.48$, $95 \% \mathrm{CI}=0.19-1.26, P=0.14)$.

\section{Sensitivity analysis}

PC patients were treated by DC-CIK immunotherapy in eight trials ${ }^{24,25,27,28,31,33-35}$ and by CIK alone in the other six trials. ${ }^{16,17,26,29,30,32}$ Studies were grouped according to different immunotherapy strategies (CIK or DC-CIK), and pooled results were compared (Table 5). The comparison showed both CIK and DC-CIK were effective in treating PC, and no obvious difference between these two methods was observed in most pooled analyses including ORR (Figure S4A), DCR (Figure S4B), and 1-year OS (Figure S5).

\section{Publication bias}

Funnel plots drawn for the studies on primary outcomes (1- and 3-year OS, ORR, and DCR) were symmetrical approximately, which indicated generally controlled publication bias and reliability of our primary conclusions (Figure 8A, 1-year OS; Figure 8B, 3-year OS; Figure 8C, ORR; Figure 8D, DCR).

\section{Discussion}

In recent years, immunotherapy using DC-CIK was found effective in PC treatment. ${ }^{16-18}$ Even though there was statistical analysis of published clinical trials, the exact therapeutic effects were not systematically evaluated and demonstrated because of sample sizes' variability among these trials. In addition, the different applied protocols in different clinical trials may lead to different clinical response. In this research, we performed an extensive online search followed by rigorous contrasting and combining data analysis in categorization, by which to provide clear and systematical conclusion.

Our analysis showed that DC-CIK immunotherapy enhanced the curative effect of chemotherapy for PC, which was supported by markedly increased ORR $(P=0.0003)$ and DCR $(P<0.00001)$ in PC patients treated by combined therapy. With the addition of DC-CIK immunotherapy, prognosis of PC patients was also improved, according to the significantly prolonged survival time (1-year OS, $P<0.00001$; 3-year OS, $P<0.00001)$ and QoL $(P=0.0009)$.

Previous study has reported the immunosuppressed status in cancer patient, and several researchers found that adjuvant immunotherapy of DC-CIK was able to enhance the efficacy of chemotherapy for various malignant tumors by reconstructing cancer patient's immune function. ${ }^{5,6}$ Our analysis showed that DC-CIK treatment can significantly improve the percentages of $\mathrm{CD}^{+}, \mathrm{CD}^{+}$, and $\mathrm{CD}^{+} \mathrm{CD}^{+} 6^{+}$ $\mathrm{T}$ cells in $\mathrm{PC}$ patients. Moreover, $\mathrm{CD} 4^{+} \mathrm{CD} 25^{+} \mathrm{CD} 127^{\text {low }}$ regulatory $\mathrm{T}$ cells negatively regulate the antitumor activity of DC-CIK immune cells ${ }^{36}$ and our analysis showed result that $\mathrm{CD} 4^{+} \mathrm{CD} 25^{+} \mathrm{CD} 127^{\text {low }}$ regulatory $\mathrm{T}$-cell subset proportion decreased after DC-CIK immunotherapy. These results indicated that immune function of chemotherapy-treated PC patients was improved after DC-CIK immunotherapy. However, no significant difference was found in $\mathrm{CD} 8^{+}$ T cells' proportion and $\mathrm{CD}^{+} / \mathrm{CD}^{+}$ratio between with and without immunotherapy, which may be caused by various choices of treatment opportunity and DC-CIK transfusion dosages in different clinical trials. ${ }^{6}$ The balance between Th1 and Th2 cells is crucial in immunotherapy. ${ }^{5}$ Our analysis showed that after DC-CIK immunotherapy, IFN- $\gamma$ (Th1

Table 5 Meta-analysis of I-year OS, ORR, and DCR in CIK and DC-CIK subgroups

\begin{tabular}{|c|c|c|c|c|c|c|c|c|c|}
\hline \multirow{2}{*}{$\begin{array}{l}\text { Immunotherapy } \\
\text { type (subgroup) }\end{array}$} & \multirow[t]{2}{*}{ Parameters } & \multicolumn{2}{|c|}{ Number of patients (n) } & \multirow{2}{*}{$\begin{array}{l}\text { Analysis } \\
\text { method }\end{array}$} & \multicolumn{2}{|c|}{ Heterogeneity } & \multirow[t]{2}{*}{ OR } & \multirow[t]{2}{*}{$95 \% \mathrm{Cl}$} & \multirow[t]{2}{*}{$P$-value } \\
\hline & & $\begin{array}{l}\text { Experimental } \\
\text { group }\end{array}$ & $\begin{array}{l}\text { Control } \\
\text { group }\end{array}$ & & $I^{2}(\%)$ & $P$-value & & & \\
\hline \multirow[t]{3}{*}{ CIK } & ORR & 186 & 248 & Fixed & 0 & 0.96 & 1.80 & I.12-2.90 & 0.01 \\
\hline & DCR & 186 & 248 & Fixed & 0 & 0.44 & 2.25 & $1.47-3.44$ & 0.0002 \\
\hline & I-Year OS & 52 & 84 & Random & 86 & 0.007 & 3.58 & $0.4 I-30.95$ & 0.25 \\
\hline \multirow[t]{3}{*}{ DC-CIK } & ORR & 142 & 142 & Fixed & 0 & 0.50 & $\mathrm{I} .57$ & $0.95-2.58$ & 0.08 \\
\hline & DCR & 142 & 142 & Fixed & 0 & 0.73 & 2.53 & I.32-4.85 & 0.005 \\
\hline & I-Year OS & 190 & 190 & Random & 0 & 0.95 & 3.62 & $2.25-5.84$ & $<0.00001$ \\
\hline
\end{tabular}

Abbreviations: DC-CIK, dendritic cells-cytokine-induced killer; DCR, disease control rate; OR, odds ratio; ORR, overall response rate; OS, overall survival. 

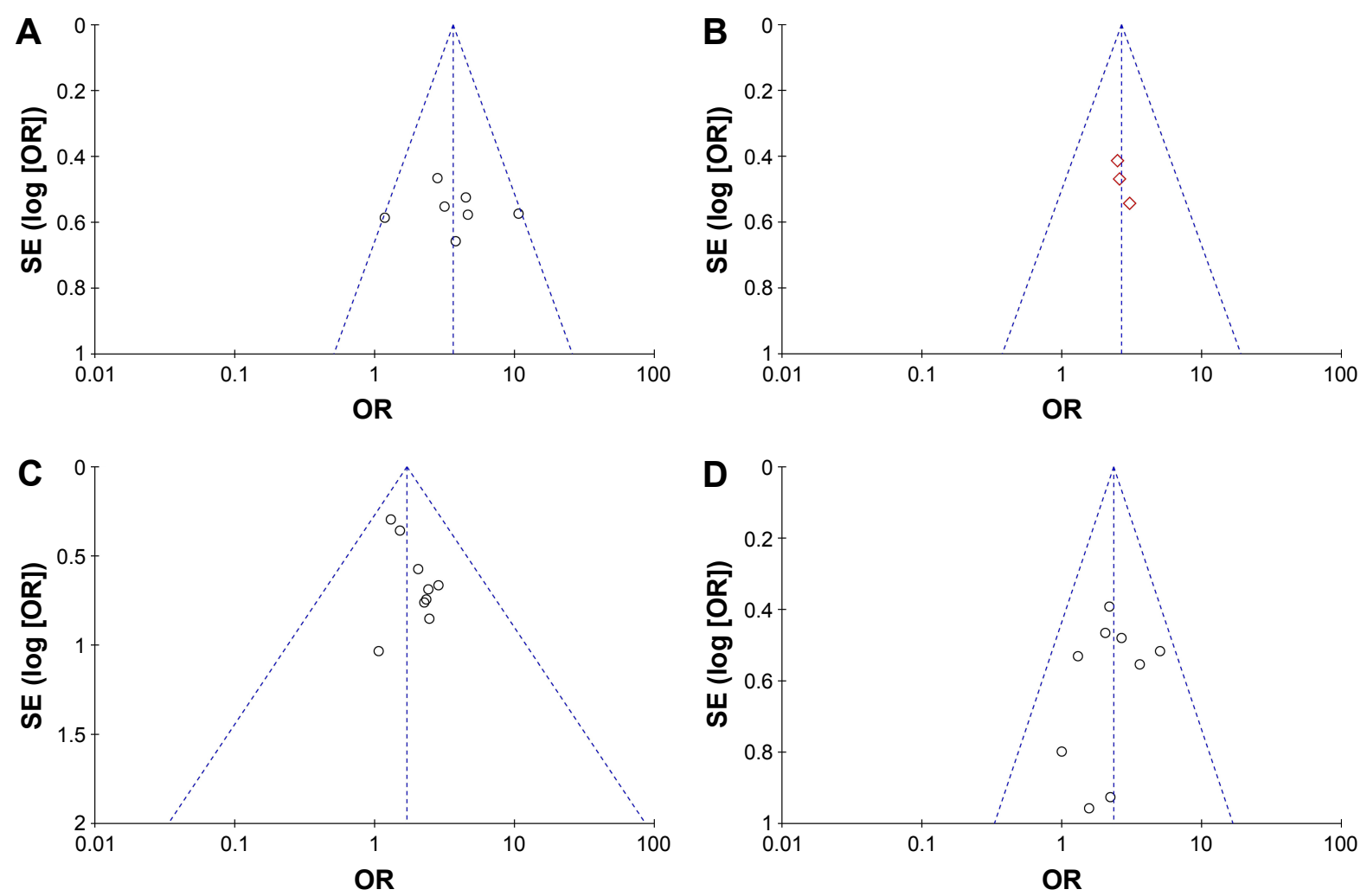

Figure 8 Funnel plot of each meta-analysis.

Notes: I-Year OS (A); 3-year OS (B); ORR (C); and DCR (D).

Abbreviations: DCR, disease control rate; ORR, overall response rate; OS, overall survival; SE, standard error; OR, odds ratio.

cytokine) level in PC patients was significantly increased, whereas IL-4 (Th2 cytokine) level was obviously decreased, indicating a passably more important role of IFN- $\gamma$ and IL-4 during the DC-CIK immunotherapy.

Safety is a crucial criterion for the popularization of clinical application of DC-CIK immunotherapy. Based on published literature up to May 2017, our meta-analysis shows that DC-CIK immunotherapy is a safe therapeutic strategy for PC, as no significant difference in adverse events was observed between with and without immunotherapy. Most side effects of DC-CIK immunotherapy were well tolerated by PC patients, and no serious adverse events or death occurred during DC-CIK therapy.

In this analysis, $\mathrm{PC}$ patients were treated by $\mathrm{DC}-\mathrm{CIK}$ immunotherapy in eight trials and CIK alone in the other six trials. To provide evidence for making the choice of using CIK or DC-CIK, difference between their therapeutic effects was evaluated by sensitivity analysis, which showed that both CIK and DC-CIK were effective in treating PC without statistical difference. These results are inconsistent with in vitro study in which DC-CIK represented higher antitumor activity than CIK alone and need to be further explored. Moreover, we conducted publication bias to verify the reliability of our result and no obvious bias exists in our primary conclusions.

\section{Limitations}

A total of 14 included trials, which met our selection criteria, turned out to be conducted on Chinese population. One trial conducted in Korea was included in our research originally but was then excluded because it lacked insufficient data. Besides, data analyzed in this research were collected from published papers rather than original patient records, which may lead to overestimation of curative effects.

\section{Conclusion}

Our meta-analysis shows that the combination of DC-CIK immunotherapy and chemotherapy is a promising immune treatment for PC patients. It markedly prolongs PC patients' survival time passably by reconstructing patients' immune function.

\section{Acknowledgments}

This study was supported by National Science Foundation of China (Nos 81201549 and 81602374), the Fundamental Research Funds for the Central Universities (No 2016qngz05), 
and the Clinical Research Award of the First Affiliated Hospital of Xi' an Jiaotong University, China (No XJTU1AFCRF-2015-011).

\section{Author contributions}

All authors contributed toward data analysis and drafting and critically revising the paper and agree to be accountable for all aspects of the work.

\section{Disclosure}

The authors report no conflicts of interest in this work.

\section{References}

1. Chen J, Guo XZ, Qi XS. Clinical outcomes of specific immunotherapy in advanced pancreatic cancer: a systematic review and meta-analysis. J Immunol Res. 2017;2017:1-16.

2. Thind K, Padrnos LJ, Ramanathan RK, Borad MJ. Immunotherapy in pancreatic cancer treatment: a new frontier. Therap Adv Gastroenterol. 2017;10(1):168-194.

3. Chen L, Zhang X. Primary analysis for clinical efficacy of immunotherapy in patients with pancreatic cancer. Immunotherapy. 2016;8(2): 223-234.

4. Chang JH, Jiang Y, Pillarisetty VG. Role of immune cells in pancreatic cancer from bench to clinical application: an updated review. Medicine (Baltimore). 2016;95(49):e5541.

5. Zhang L, Mu Y, Zhang A, et al. Cytokine-induced killer cells/dendritic cells-cytokine induced killer cells immunotherapy combined with chemotherapy for treatment of colorectal cancer in China: a metaanalysis of 29 trials involving 2,610 patients. Oncotarget. 2017;8(28): 45164-45177.

6. Zhou C, Liu D, Li J, et al. Chemotherapy plus dendritic cells co-cultured with cytokine-induced killer cells versus chemotherapy alone to treat advanced non-small-cell lung cancer: a meta-analysis. Oncotarget. 2016;7(52):86500-86510.

7. Dillman RO, Duma CM, Ellis RA, et al. Intralesional lymphokineactivated killer cells as adjuvant therapy for primary glioblastoma. J Immunother. 2009;32(9):914-919.

8. Yamaguchi Y, Hihara J, Hironaka K, et al. Postoperative immunosuppression cascade and immunotherapy using lymphokine-activated killer cells for patients with esophageal cancer: possible application for compensatory anti-inflammatory response syndrome. Oncol Rep. 2006;15(4):895-901.

9. Yang Y, Lim O, Kim TM, et al. Phase I study of random healthy donor-derived allogeneic natural killer cell therapy in patients with malignant lymphoma or advanced solid tumors. Cancer Immunol Res. 2016;4(3):215-224.

10. Zhao Z, Liao H, Ju Y. Effect of compound Kushen injection on T-cell subgroups and natural killer cells in patients with locally advanced nonsmall-cell lung cancer treated with concomitant radiochemotherapy. $J$ Tradit Chin Med. 2016;36(1):14-18.

11. Dudley ME, Gross CA, Somerville RP, et al. Randomized selection design trial evaluating CD8+-enriched versus unselected tumorinfiltrating lymphocytes for adoptive cell therapy for patients with melanoma. J Clin Oncol. 2013;31(17):2152-2159.

12. Kageyama S, Ikeda H, Miyahara $Y$, et al. Adoptive transfer of MAGE-A4 $\mathrm{T}$-cell receptor gene-transduced lymphocytes in patients with recurrent esophageal cancer. Clin Cancer Res. 2015;21(10):2268-2277.

13. Mu Y, Zhou CH, Chen SF, et al. Effectiveness and safety of chemotherapy combined with cytokine-induced killer cell/dendritic cellcytokine-induced killer cell therapy for treatment of gastric cancer in China: a systematic review and meta-analysis. Cytotherapy. 2016;18(9): 1162-1177.
14. Wang ZX, Cao JX, Wang M, et al. Adoptive cellular immunotherapy for the treatment of patients with breast cancer: a meta-analysis. Cytotherapy. 2014;16(7):934-945.

15. Liu Y, Mu Y, Zhang A, et al. Cytokine-induced killer cells/dendritic cells and cytokine-induced killer cells immunotherapy for the treatment of esophageal cancer in China: a meta-analysis. Onco Targets Ther. 2017;10:1897-1908.

16. Wang M, Shi SB, Qi JL, Tang XY, Tian J. S-1 plus CIK as secondline treatment for advanced pancreatic cancer. Med Oncol. 2013; 30(4):747.

17. Wang Z, Liu Y, Li R, et al. Autologous cytokine-induced killer cell transfusion increases overall survival in advanced pancreatic cancer. J Hematol Oncol. 2016;9(1):6.

18. Chung MJ, Park JY, Bang S, Park SW, Song SY. Phase II clinical trial of ex vivo-expanded cytokine-induced killer cells therapy in advanced pancreatic cancer. Cancer Immunol Immunother. 2014;63(9): 939-946.

19. Li W, Wang Y, Kellner DB, Xu L, Mai L. Efficacy of cytokine-induced killer cells in the treatment of elderly patients with metastatic pancreatic adenocarcinoma. Cent Eur J Immunol. 2015;40(2):188-193.

20. Zeng X, Zhang Y, Kwong JS, et al. The methodological quality assessment tools for preclinical and clinical studies, systematic review and meta-analysis, and clinical practice guideline: a systematic review. J Evid Based Med. 2015;8(1):2-10.

21. Han RX, Liu X, Pan P, Jia YJ, Yu JC. Effectiveness and safety of chemotherapy combined with dendritic cells co-cultured with cytokineinduced killer cells in the treatment of advanced non-small-cell lung cancer: a systematic review and meta-analysis. PLoS One. 2014;9(9): e108958.

22. Jackson D, White IR, Riley RD. Quantifying the impact of betweenstudy heterogeneity in multivariate meta-analyses. Stat Med. 2012; 31(29):3805-3820.

23. Cai XR, Li X, Lin JX, et al. Autologous transplantation of cytokineinduced killer cells as an adjuvant therapy for hepatocellular carcinoma in Asia: an update meta-analysis and systematic review. Oncotarget. 2017;8(19):31318-31328.

24. Ge JN, Ge CL. Effect of DC-CIK cells on immune function in patients with pancreatic cancer after chemotherapy. J Community Med. 2016; 14(17):26-27.

25. Kang J, Zhang HY. Clinical analysis of gemcitabine chemotherapy coast CIK cell treatment of pancreatic cancer. China Contin Med Educ. 2016;8(5):141-142.

26. Li XD. Clinical observation of three dimensional conformal radiotherapy concurrent with chemotherapy combined with autologous cytokine induced killer cell immunotherapy for treatment of locally advanced pancreatic cancer. China Clin Prac Med. 2016;7(2):64-66.

27. Liu AH. Influence of chemotherapy combined with DC-CIK cell on the immune function in patients with pancreatic cancer. Chin J Clin. 2012;6(20):6301-6305.

28. Mu L, Zhong XP, Liu HM. Clinical efficacy evaluation of DC-CIK in the treatment of advanced pancreatic cancer after chemotherapy. Guizhou Med J. 2016;40(5):532-535.

29. Shen D, Liu T, Lin QF, et al. Clinical evaluation of cytokine-induced killer cells combination with chemotherapy in patients with advanced pancreatic cancer. J Prac Med. 2015;31(13):2148-2151.

30. Wang J. Effects of gemcitabine chemotherapy combined with CIK cells therapy in the treatment of pancreatic cancer. Med Pharm Yunnan. 2015;36(2):183-185.

31. Wen QX, Zhu Y, Xu B, Wang MF. The study of clinical application of DC-CIK combined with chemotherapy on advanced pancreatic cancer. Mod J Integr Trad Chin West Med. 2013;22(36):4065-4066.

32. Zhang YH, Wang MH, Tian Y, Xu F. Clinical research of patients with advanced pancreatic cancer treated with CIK cells combined with chemotherapy. J Basic Clin Oncol. 2013;26(4):317-319.

33. Zhang YL. Study of the influence on immunologic balance and clinical effect by chemotherapy combined with DC-CIK cell to patients with pancreatic cancer. China J Mod Med. 2014;24(9):42-45. 
34. Zhang ZQ, Wang ZY, Zheng S, Wang XZ, He LJ. Effect of DC-CIK cell therapy on immune function and clinical efficacy analysis of DC-CIK for treatment of pancreatic cancer after chemotherapy. China Prac Med. 2016;11(7):183-184.

35. Zheng C, Yin YL, Jin HF, Liu HQ. Effect of chemotherapy combined with DC-CIK cell therapy on immune function in patients with pancreatic cancer. Bao Jian Wen Hui. 2016;6:153.
36. Liu H, Li J, Wang F, et al. Comparative study of different procedures for the separation of peripheral blood mononuclear cells in cytokineinduced killer cell immunotherapy for hepatocarcinoma. Tumour Biol. 2015;36(4):2299-2307. 


\section{Supplementary materials}

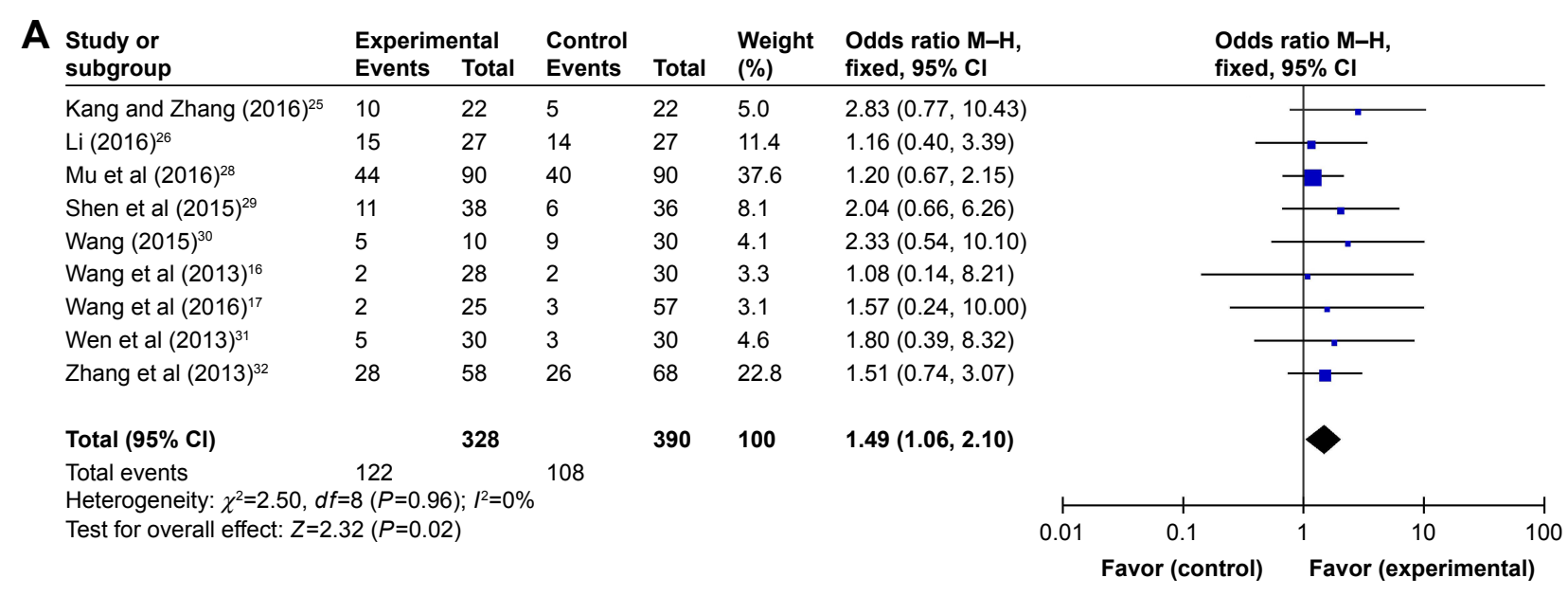

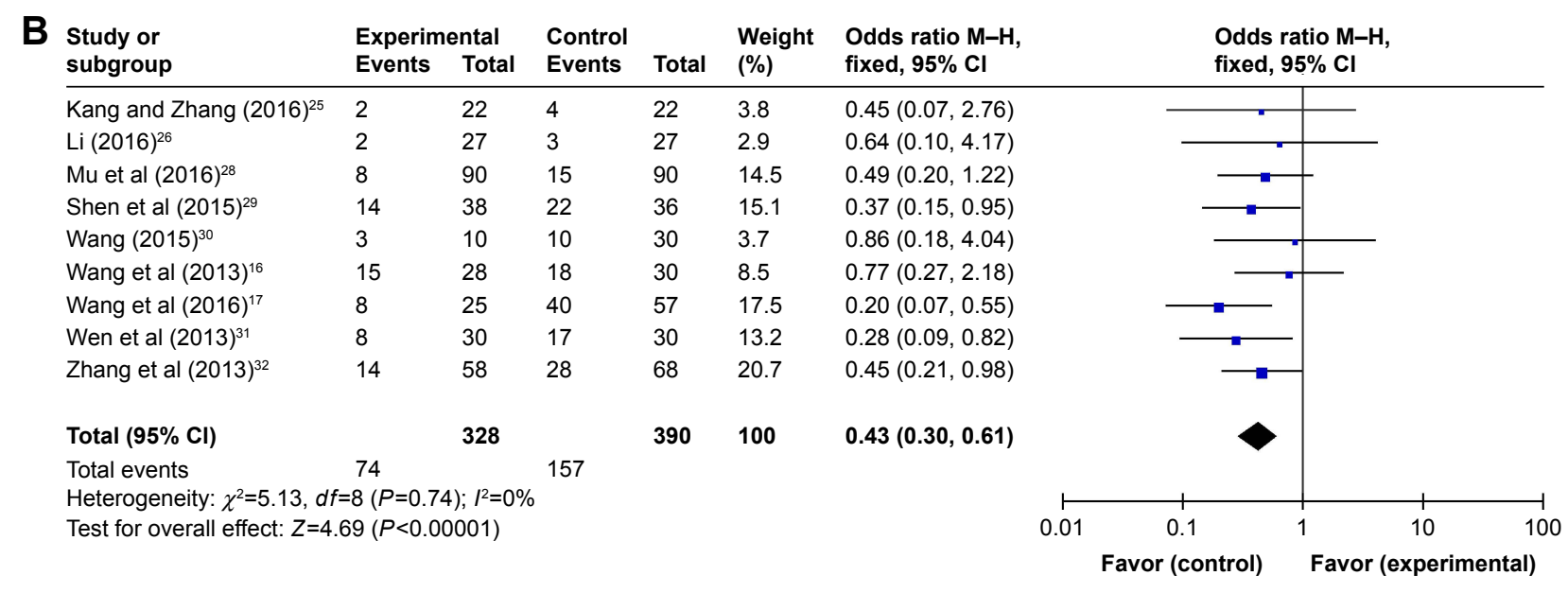

Figure SI (Continued) 


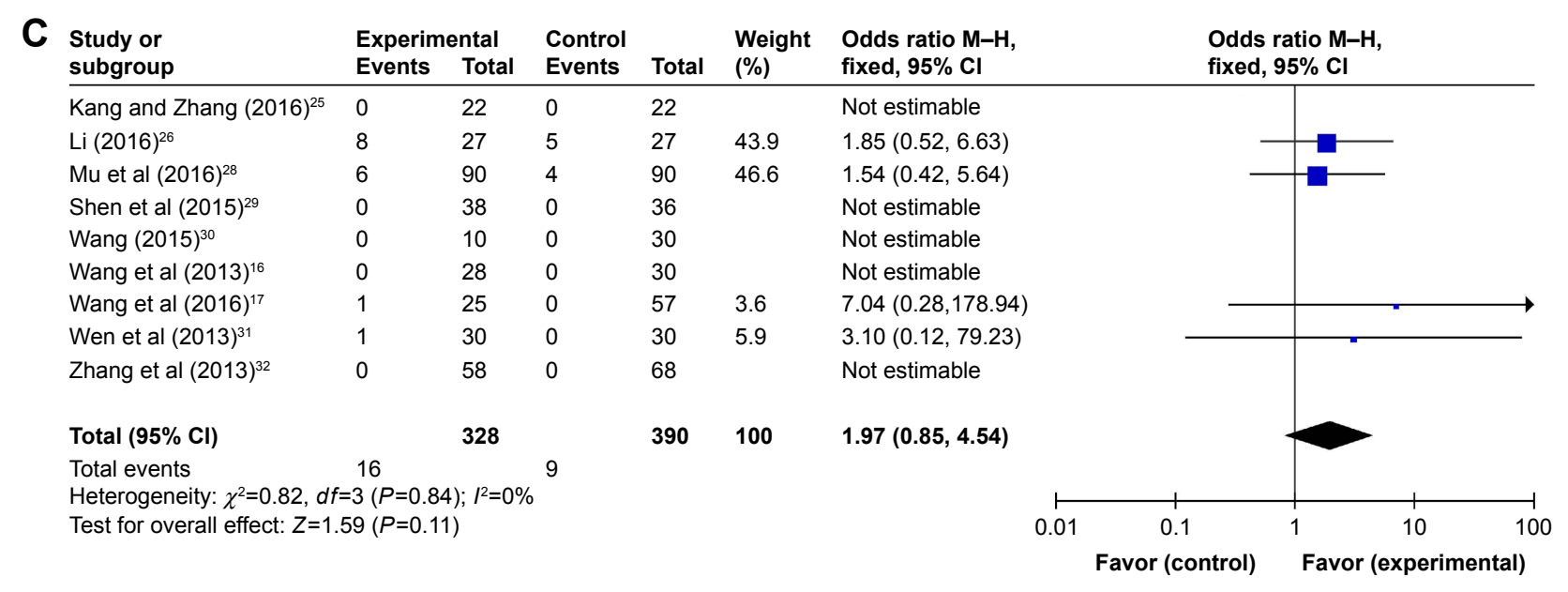

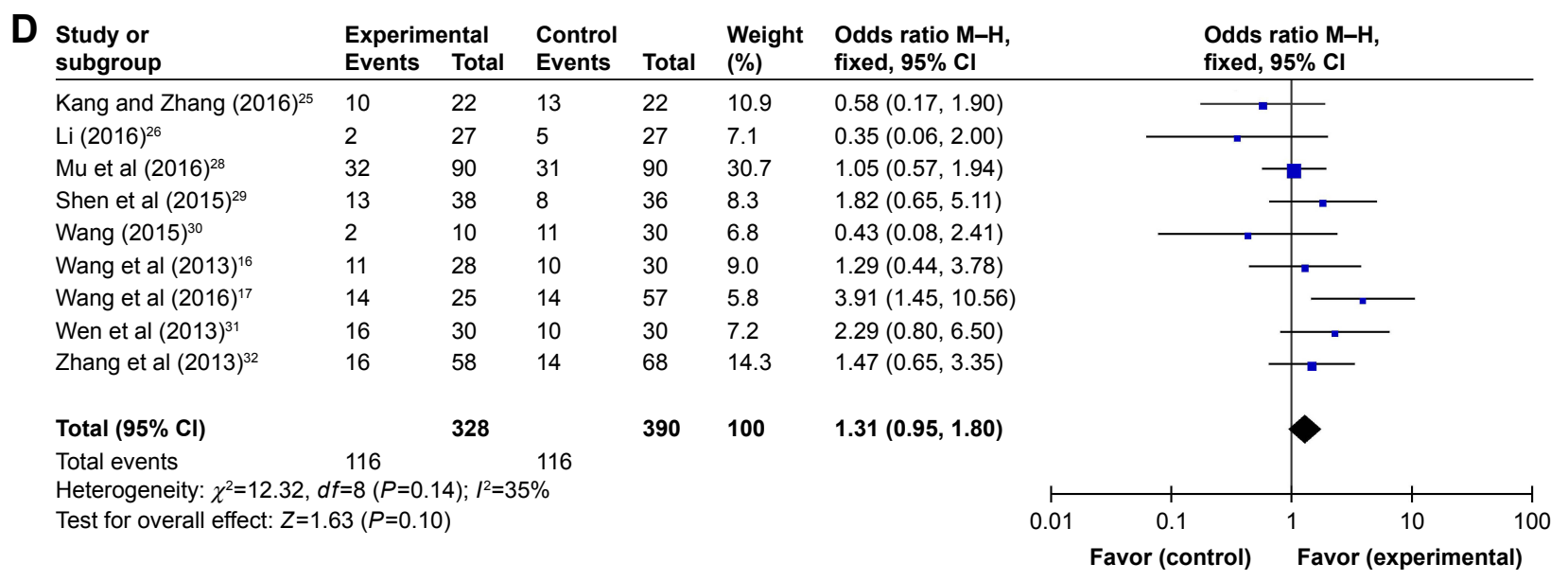

Figure SI Forest plots of the comparison of PR (A), PD (B), CR (C), and SD (D) rates between the experimental and control groups.

Notes: Control group, chemotherapy alone group; experimental group, chemotherapy with DC-CIK immunotherapy. The fixed-effects meta-analysis model (M-H method) was used.

Abbreviations: $\mathrm{Cl}$, confidence interval; $\mathrm{CR}$, complete response; CIK, cytokine-induced killer; DC-CIK, dendritic cells-CIK; M-H, Mantel-Haenszel; PD, progressive disease; PR, partial response; SD, stable disease. 


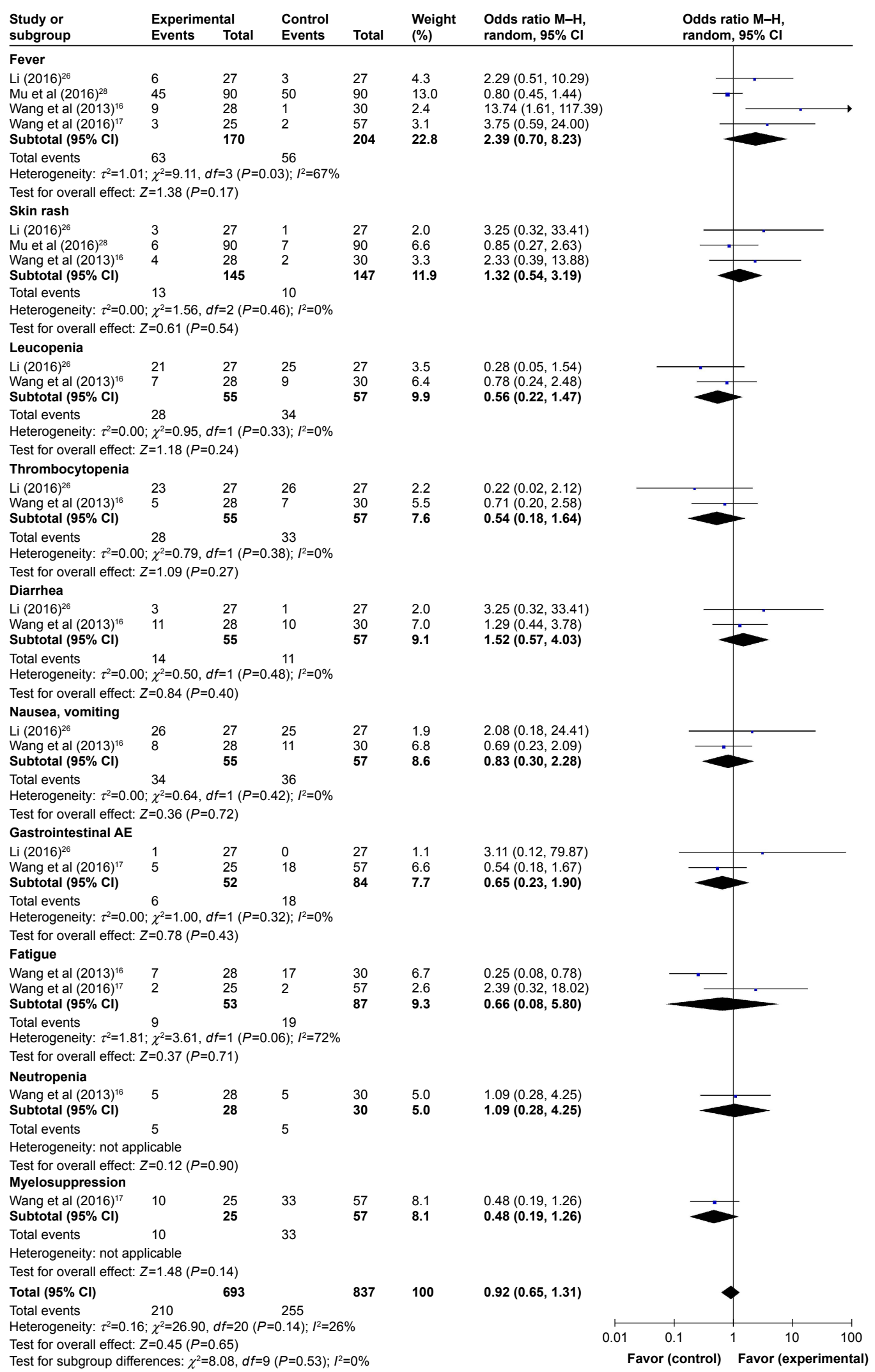

Figure S2 Forest plot of the comparison of adverse effects between the experimental and control groups.

Notes: Control group, chemotherapy alone group; experimental group, chemotherapy with DC-CIK immunotherapy. The random effects meta-analysis model ( $\mathrm{M}-\mathrm{H}$ method) was used.

Abbreviations: $\mathrm{Cl}$, confidence interval; DC-CIK, dendritic cells-cytokine-induced killer; $\mathrm{M}-\mathrm{H}$, Mantel-Haenszel. 


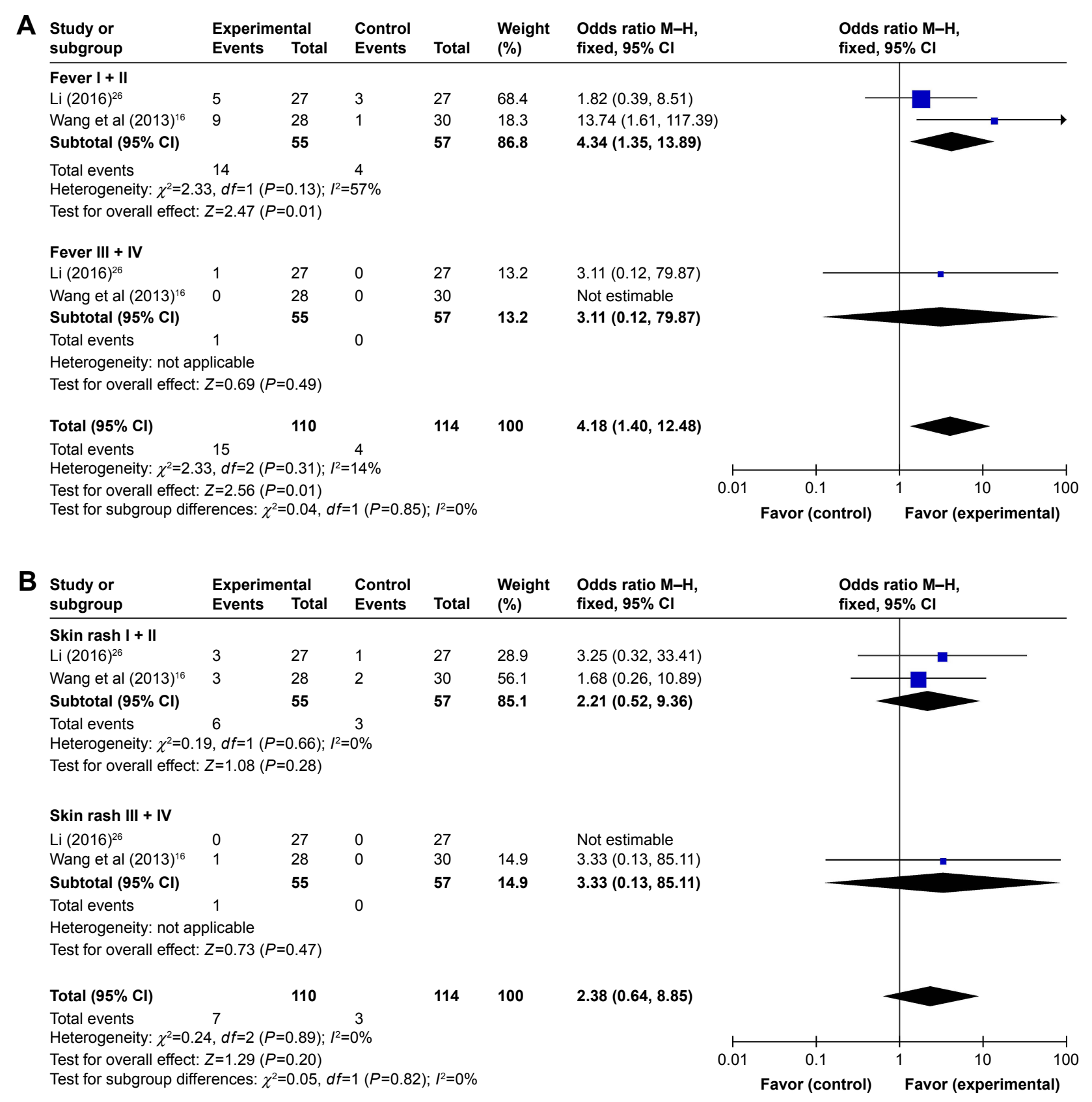

Figure S3 (Continued) 


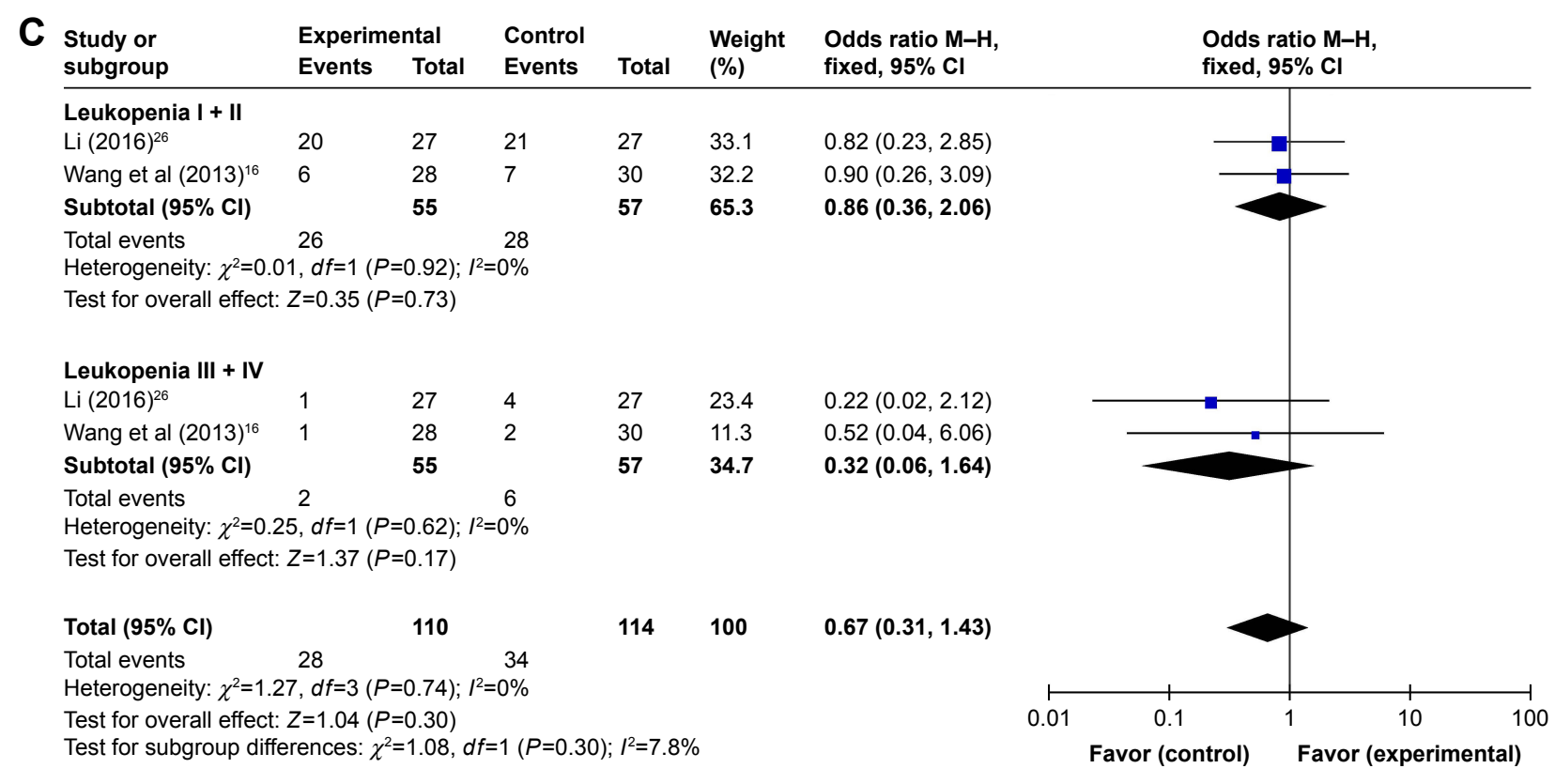

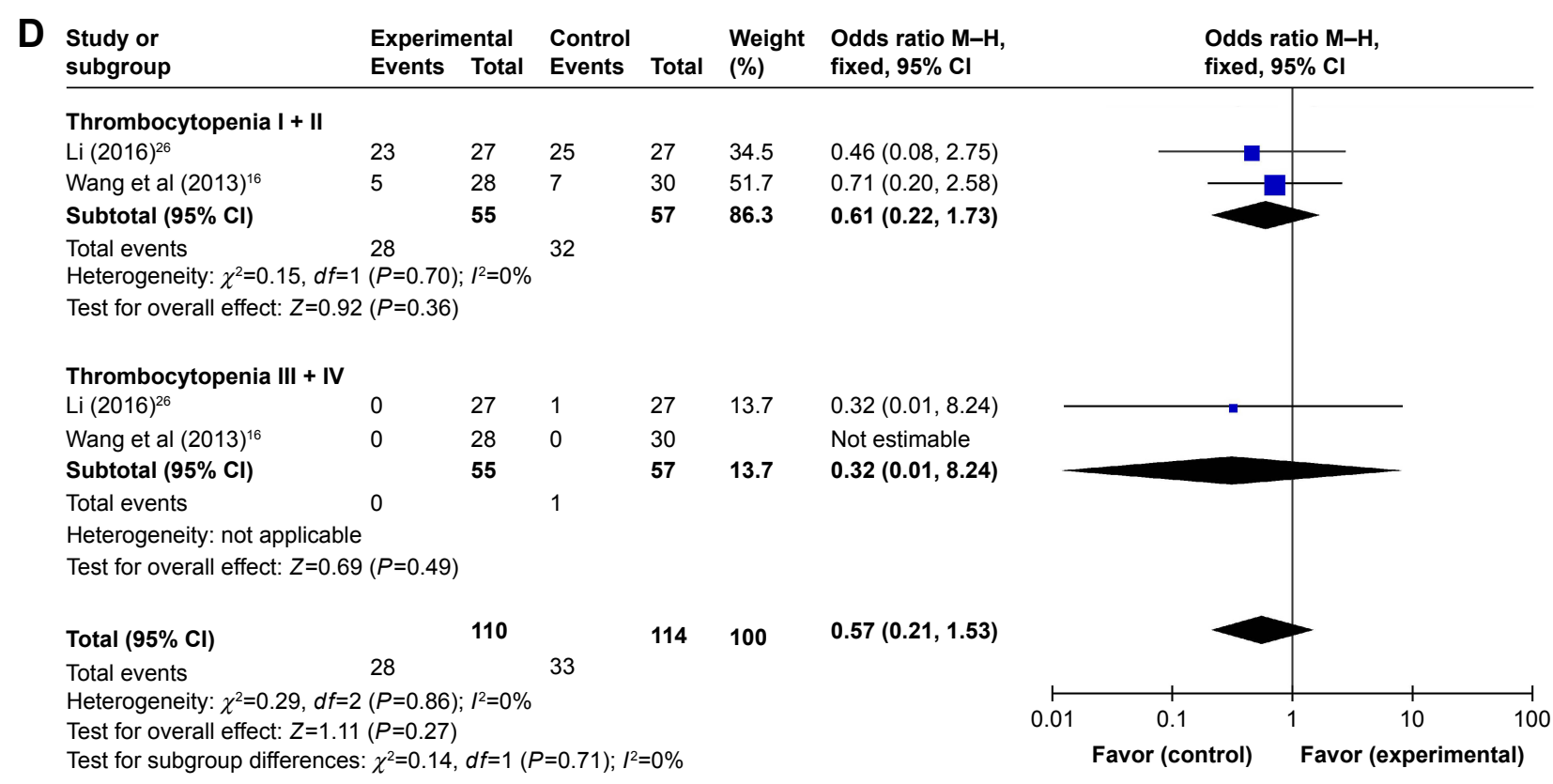

Figure S3 (Continued) 


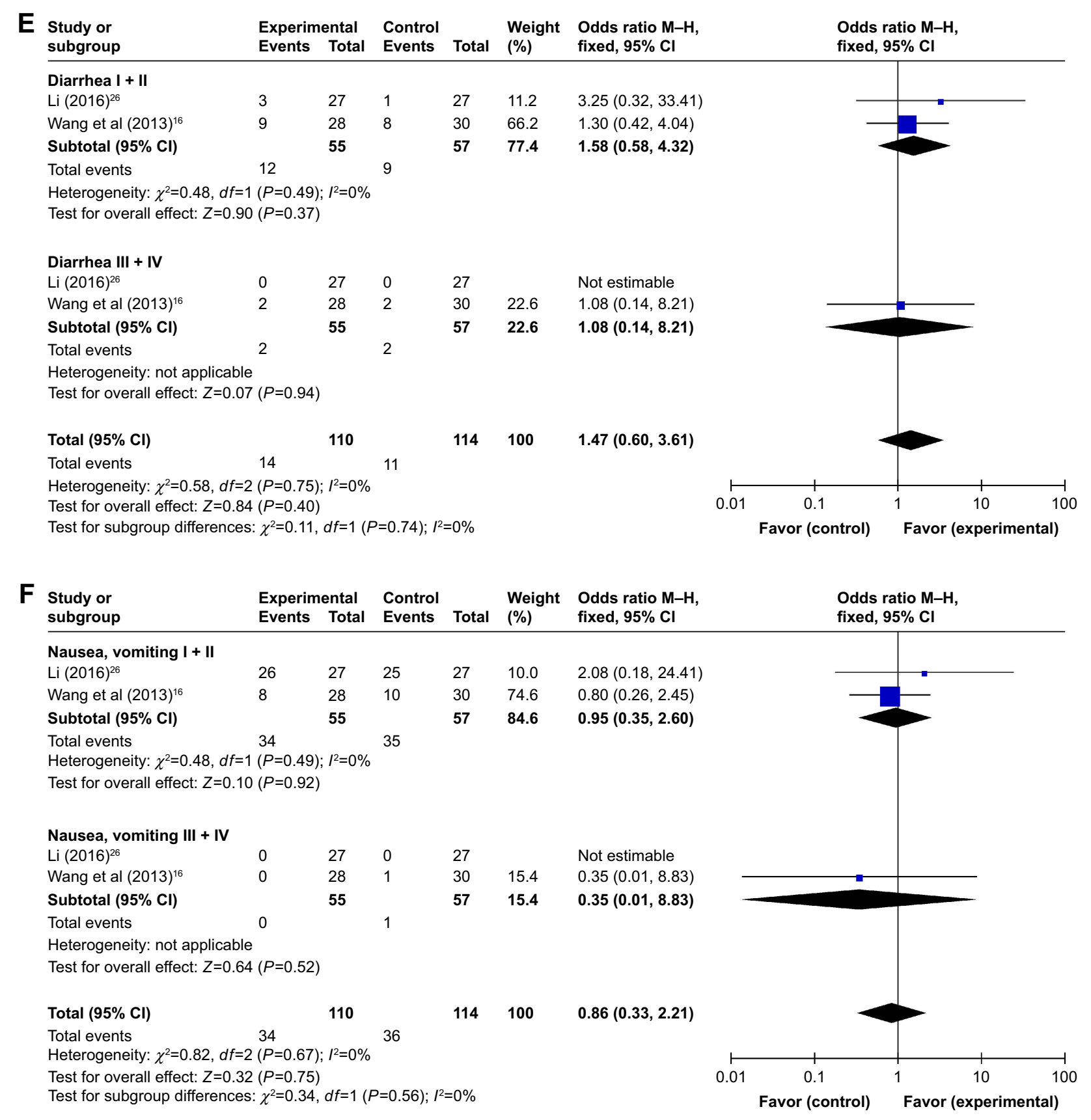

Figure S3 Forest plots of the comparison of all-grade adverse effects including fever (A), skin rash (B), leukopenia (C), thrombocytopenia (D), diarrhea (E), and nausea and vomiting $(\mathbf{F})$.

Notes: Control group, chemotherapy alone group; experimental group, chemotherapy with DC-CIK immunotherapy. The fixed-effects meta-analysis model (M-H method) was used.

Abbreviations: $\mathrm{Cl}$, confidence interval; DC-CIK, dendritic cells-cytokine-induced killer; $\mathrm{M}-\mathrm{H}$, Mantel-Haenszel. 


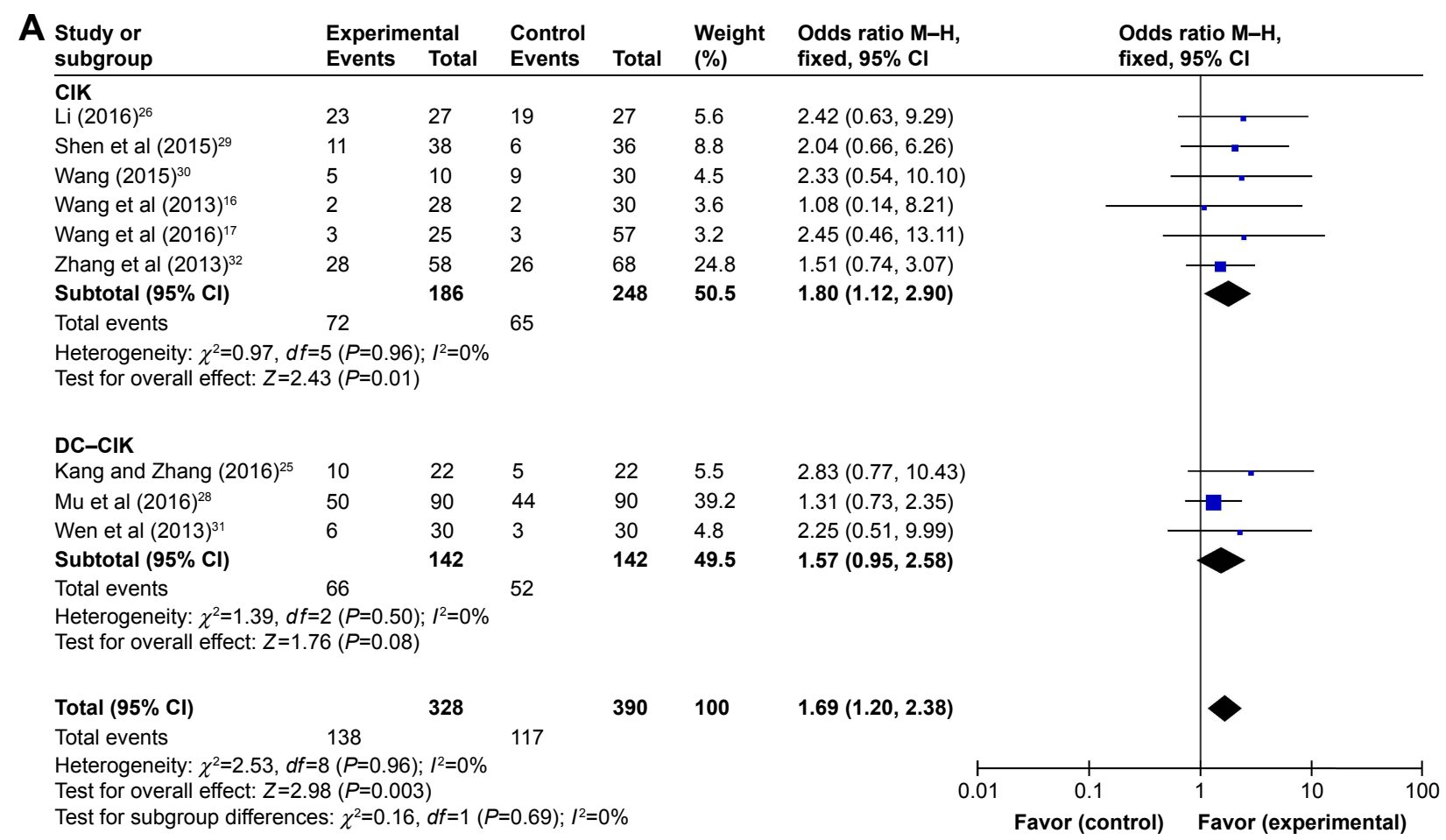

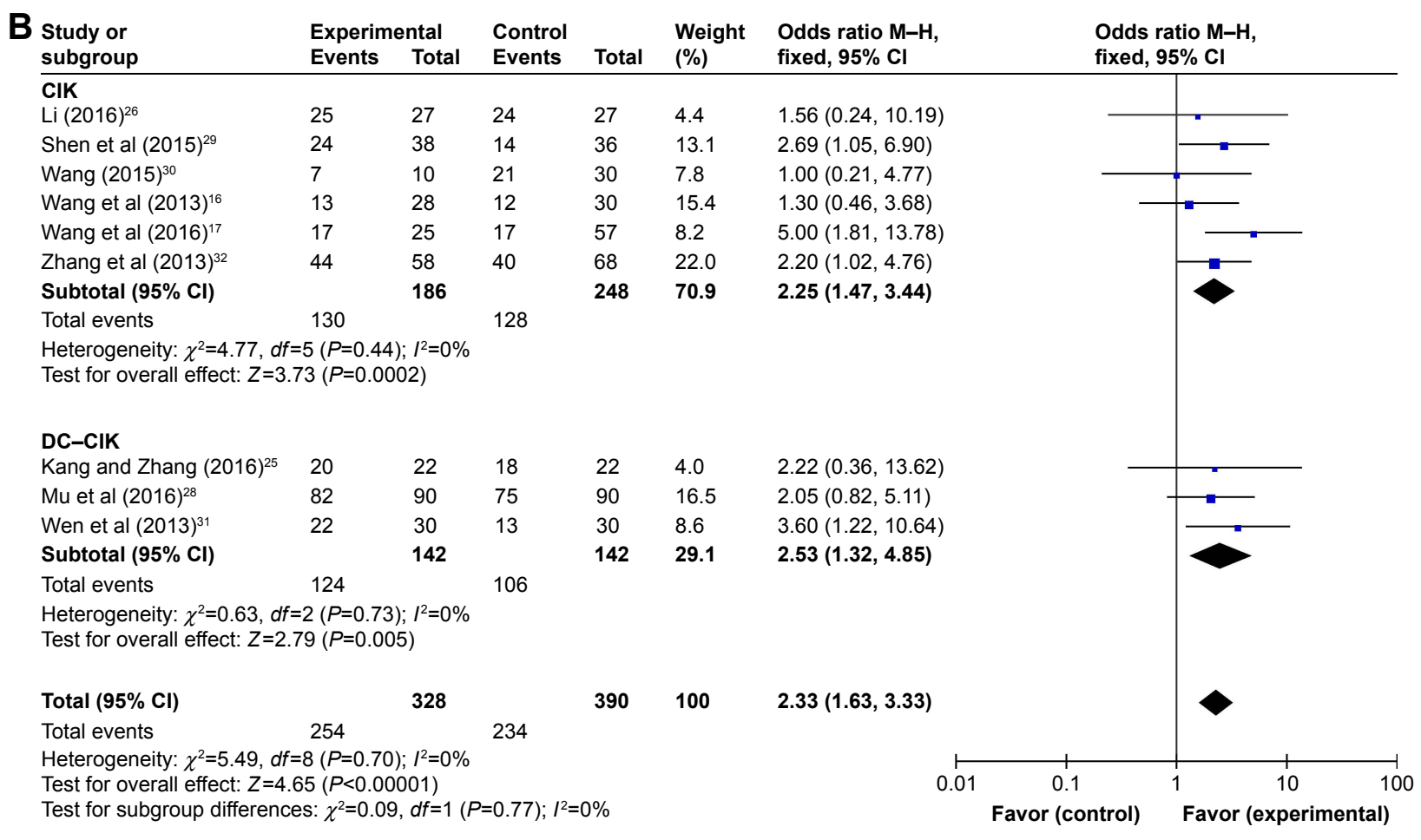

Figure S4 Forest plots of the comparison of ORR (A) and DCR (B) in CIK and DC-CIK subgroups.

Notes: Control group, chemotherapy alone group; experimental group, chemotherapy with DC-CIK immunotherapy. The fixed-effects meta-analysis model (M-H method) was used.

Abbreviations: $\mathrm{Cl}$, confidence interval; CIK, cytokine-induced killer; DC-CIK, dendritic cells-CIK; DCR, disease control rate; M-H, Mantel-Haenszel; ORR, overall response rate. 


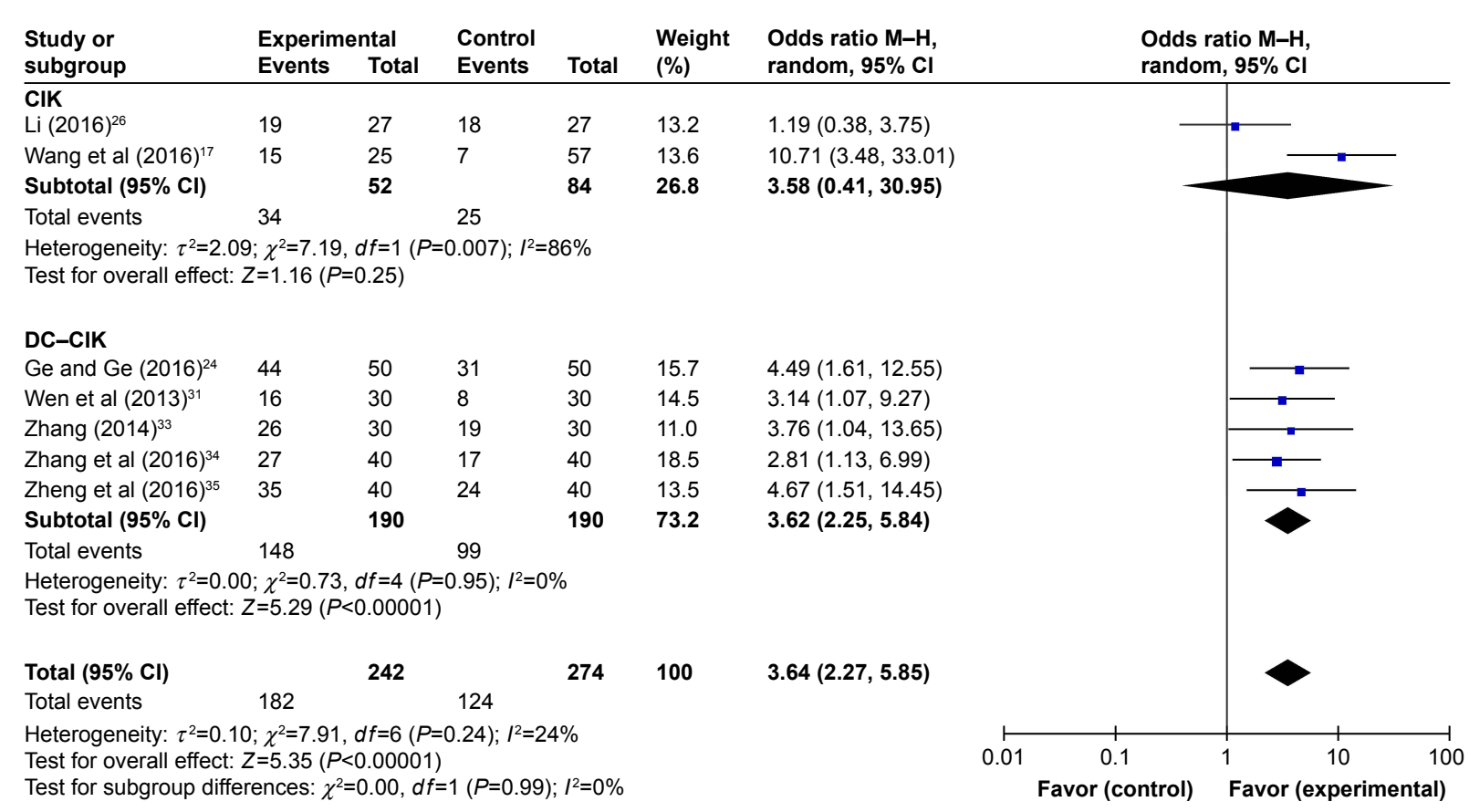

Figure S5 Forest plot of the comparison of I-year OS in CIK and DC-CIK subgroups.

Notes: Control group, chemotherapy alone group; experimental group, chemotherapy with DC-CIK immunotherapy. The random effects meta-analysis model (M-H method) was used.

Abbreviations: $\mathrm{Cl}$, confidence interval; CIK, cytokine-induced killer; DC-CIK, dendritic cells-CIK; M-H, Mantel-Haenszel; OS, overall survival.

\section{Publish your work in this journal}

OncoTargets and Therapy is an international, peer-reviewed, open access journal focusing on the pathological basis of all cancers, potential targets for therapy and treatment protocols employed to improve the management of cancer patients. The journal also focuses on the impact of management programs and new therapeutic agents and protocols on

\section{Dovepress}

patient perspectives such as quality of life, adherence and satisfaction. The manuscript management system is completely online and includes a very quick and fair peer-review system, which is all easy to use. Visit http://www.dovepress.com/testimonials.php to read real quotes from published authors. 